

\title{
Fabrication-aware shape parametrisation for the structural optimisation of shell structures
} Romain Mesnil, Cyril Douthe, Christiane Richter, Olivier Baverel

\section{To cite this version:}

Romain Mesnil, Cyril Douthe, Christiane Richter, Olivier Baverel. Fabrication-aware shape parametrisation for the structural optimisation of shell structures. Engineering Structures, 2018, 176, pp.569 584. 10.1016/j.engstruct.2018.09.026 . hal-01899204

\section{HAL Id: hal-01899204 https://hal.science/hal-01899204}

Submitted on 23 Oct 2018

HAL is a multi-disciplinary open access archive for the deposit and dissemination of scientific research documents, whether they are published or not. The documents may come from teaching and research institutions in France or abroad, or from public or private research centers.
L'archive ouverte pluridisciplinaire HAL, est destinée au dépôt et à la diffusion de documents scientifiques de niveau recherche, publiés ou non, émanant des établissements d'enseignement et de recherche français ou étrangers, des laboratoires publics ou privés. 


\title{
Fabrication-aware shape parametrisation for the structural optimisation of shell structures
}

\author{
Romain Mesnil $^{\mathrm{a}, \mathrm{b}, *}$, Cyril Douthe ${ }^{\mathrm{a}}$, Christiane Richter ${ }^{\mathrm{a}}$, Olivier Baverel ${ }^{\mathrm{a}}$ \\ ${ }^{a}$ Laboratoire Navier, UMR 8205, École des Ponts, IFSTTAR, CNRS, UPE, Marne-La-Vallée, France \\ ${ }^{b}$ Bouygues Construction SA, Guyancourt, France
}

\begin{abstract}
The difficulty to construct mechanically optimal shells may limit the use of structural optimisation in practice. The objective of this paper is to propose a new parametric representation of doubly curved shapes suited for structural optimisation of architectural shells that inherently considers fabrication constraints. We focus on a common construction constraint: the covering of building envelopes with planar facets. This paper proposes to implement the so-called marionette technique as a Computer-Aided-Design tool that guarantees covering of free-form shapes with planar quadrilateral facets.

General considerations on the size and nature of the optimisation space created with this method are made. It is demonstrated through different case-studies that the quality of the parametrisation for shape optimisation of shell structures is similar to the one offered by Bézier surfaces, an ubiquitous modelling technique. The proposed method conciliates thus fabrication and structural performance.
\end{abstract}

Keywords: Shape optimization, Shell structures, Free-form architecture, Fabrication-aware design, PQ-Mesh, NURBS, Marionette method

\section{Introduction}

\subsection{Motivation}

Structural optimisation is a powerful tool that aims at minimising some functional describing the mechanical behaviour of a structure. It has been popularised in architecture by the concept of form-finding by optimisation for shell structures [1]. This approach considers the shape of a shell structure as the result of a structural optimisation process. It shares some similarities with mechanical form-finding approaches, which have been used since the introduction of the force density method for the computation of tensile structures [2]. Optimisation overcomes the limitations of form-finding methods, because it can consider different load cases or non-linear analysis [3].

Despite numerous contributions to the research on shape optimisation of shell structures, applications to the construction industry remain scarce [4]. For Borgart [5], one of the main limiting factors for practical application of optimisation is that a structurally optimal design is not necessarily constructible. The construction of shell structures in architecture is indeed subject to strong economical constraints linked to fabrication, as well as architectural imperatives. Fabrication constraints are particularly demanding when doubly curved shells are considered, as

\footnotetext{
* Corresponding author

E-mail: romain.mesnil@enpc.fr
}

many properties of the constitutive elements of the building (facets planarity, rational offset of the structural layout, repetition of elements) are lost.

A structural optimisation problem consists of three aspects: the first one, the geometrical definition, is directly related to the constructibility of the structure. The second aspect is the mechanical modelling, which sets the objective functions, the boundary conditions and the type of analysis. The last aspect is the mathematical solution of the optimisation problem, which involves the selection of the proper numerical scheme, either based on gradient descent or meta-heuristic. This article focuses thus on the aspect of geometrical definition for structural optimisation and proposes the definition of design spaces with embedded fabrication constraint for shape optimisation. In the following, the authors consider the covering of surfaces with planar quadrangles to be the main fabrication constraint. This constraint has been identified in previous research on glazed gridshells [6,7], and it is of interest for the rationalisation of formwork for concrete shells. Other fabrication related to the surface parameterisation, like the aspect ratio of the panels or the size of their bounding box should be considered in practice.

\subsection{Previous work on fabrication-aware structural optimi- sation}

Precedent of fabrication-driven design of shells or gridshells include most of the work of master builders from the twentieth century. The definition of analytical shapes 
allowed them to build a deep understanding of the doublycurved shells they were constructing before computeraided design methods were available [8]. Franz Dischinger [9] designed thin shells described by surfaces of revolution, for which he developed analytical solutions. Felix Candela is well-known for his shells based upon ruled surfaces [10]. More recently, Jörg Schlaich, Hans Schober [11] and their team designed glass-covered gridshells based upon surfaces of translation.

Fabrication-aware design used in the context of structural optimisation is less common. Structural optimisation of gridshells covering surfaces of translations was performed by [12, 13], but the design space offered by those shapes remains quite restricted. The same can be inferred for other families of surfaces covered by planar facets, like Monge surface [14] or canal-surfaces. Fujita and Ohsaki[15] considered the optimisation of shell by imposing constraints on surfaces invariants (for example a zero gaussian curvature and developable surfaces). This point of view is different from the one adopted in architectural geometry, which considers that surface parametrisation is the key problem to be addressed for construction rationality. Moreover, engineers generally aim at introducing double curvature in shells to increase their stiffness, so that the design space offered by developable surfaces can be considered to be suboptimal for structural optimisation. The integration of conjugate curve networks can also be performed on a surface found by the means of structural optimisation, as proposed in [16]. However, the topology of the network of lines of curvatures is not known beforehand, which complicates the dialogue between contractors, engineers and architects. Furthermore, many structural systems based on quadrilateral patterns are orthotropic $[17,18]$ : shape optimisation using shell elements can be generalised to discrete periodic structural patterns, but require to respect local symmetries of the parameterisation.

The generation of a large design space with planar facets suited for structural optimisation is thus an issue that remains to be further explored.

\subsection{Previous work on parametrisation of shape optimisa- tion for shells}

The description of the geometrical design space is a crucial step for the optimisation process, even when fabrication constraints are not considered. Appropriate description of parametric space for the structural optimisation of shells is an active topic research. Different approaches are used in recent research: node-based optimisation, CADbased optimisation, linear combination of mode shapes, etc.

In node-based optimisation approaches, the coordinates of each node is seen as a design variable. It offers the broadest design space, but the results become mesh dependent. Regularisation methods must be employed, examples of such techniques can be found in [19], [20] and [21].
In CAD-based parametrisation, the parameters controlling the CAD become the design variables. This restricts the size of the design space and makes the computation more efficient. The reduced number of variables can however make the solution suboptimal. This approach was introduced by Braibant and Fleury [22] and has been successfully used, for example for the maximisation of stiffness of shell structures under self-weight by [23]. The development of isogeometric analysis, which was proposed by Hughes [24], has considerably enriched this field of study, as integration of equations of elasticity can be performed on curved elements. Isogeometric analysis generally outperforms classical finite element analysis, and was adapted both to NURBS and T-splines [25].

\subsection{Research objectives}

As geometries are often generated with CAD tools, CAD-based optimisation can naturally be used in the context of real-life projects. The exploration of the design space is also eased by the lower number of variables, which is interesting when structural optimisation is used at early stages of design or for multiple optimisation criteria. Nonuniform rational basis splines (NURBS) are commonly used for shape modelling, but they do not take into account basic fabrication constraints. Their extension, Tsplines, suffer from the same limitations.

The purpose of this paper is therefore to introduce a CAD-based shape generation framework integrating the constraint of facets planarity. Practical applications are the rationalisation of formworks for reinforced concrete shells, but also steel glazed gridshells. The proposed method uses NURBS and Bézier splines, but is not a subspace of NURBS. It is therefore not possible to conclude on its suitability to structural optimisation a priori.

The proposed parametrisation method is applied to the structural optimisation of a shell on three supports inspired by the Kresge Auditorium at MIT and by the roof of the French National Centre for Innovation and Technology (CNIT), the largest spanning concrete shell to this day. This shape has been used as a benchmark in numerous studies, including [26, 23, 27]. A dome supported on an elliptic boundary inspired by [3] is also studied. Different load cases and performance metrics are considered.

The first Section introduced relevant literature on shape optimisation in architecture and an overview of fabrication-aware design. The second Section presents a new method for shape generation with planar facets as well as the general methodology for fabrication-aware design space exploration. The third Section presents a study for shells supported on three supports. Design guidelines and assessment of the results are then proposed.

\section{Methodology}

\subsection{Mathematical background of structural optimisation}

A typical optimisation problem is written as the minimisation of an objective function $f$ for a given set of design 
variables $\mathbf{x}$ under design constraints. The mathematical definition follows:

$$
\min _{\mathbf{x}} f(\mathbf{x})
$$

subject to:

$$
\begin{array}{cr}
\forall i \in\left[1 ; n_{e}\right] & g_{i}(\mathbf{x})=0 \\
\forall i \in\left[1 ; n_{i}\right] & h_{i}(\mathbf{x}) \geq 0 \\
\forall i \in[1 ; d] & x_{i}^{L} \leq x_{i} \leq x_{i}^{U}
\end{array}
$$

The minimisation is usually done under equality and inequality constraints. The design variables are in a bounded domain, for the $\mathrm{i}^{\text {th }}$ design variable, $x_{i}^{L}$ is the lower bound and $x_{i}^{U}$ is the upper bound. In equation (1) he number $d$ is the number of design variables, later called dimension of the design space in the followings of this paper; $n_{e}$ is the number of equality constraints and $n_{i}$ is the number of inequality constraints.

In the followings of this paper, the function $f$ to minimise is either the maximal displacement $(\delta)$ or the strain energy $\mathcal{E}$ under a given load, or the opposite of the minimal buckling load $\left(-p_{c r}\right)$. Strain energy is a well-known metric in structural optimisation, for mathematical and physical reasons: this quantity considers both stress and strain and is more regular than a performance metric based on maximal displacements. However, in practice, building codes impose deflection criteria based on the maximal displacements. A constrained optimisation problem solved with the penalty method would include a function of the maximal displacement so that the unconstrained optimisation of displacement solved in this article gives some insight on its solution.

Shapes generated with the marionette technique satisfy exact facet planarity, which corresponds to an equality constraint. For any quadrilateral facet $F^{i}$, with vertices $\left(\mathbf{F}_{\mathbf{0}}^{\mathbf{i}}, \mathbf{F}_{\mathbf{1}}^{\mathbf{i}}, \mathbf{F}_{\mathbf{2}}^{\mathbf{i}}, \mathbf{F}_{\mathbf{3}}^{\mathbf{i}}\right)$, the facet planarity reads as:

$$
\operatorname{det}\left(\mathbf{F}_{\mathbf{1}}^{\mathbf{i}}-\mathbf{F}_{\mathbf{0}}^{\mathbf{i}}, \mathbf{F}_{\mathbf{2}}^{\mathbf{i}}-\mathbf{F}_{\mathbf{0}}^{\mathbf{i}}, \mathbf{F}_{\mathbf{3}}^{\mathbf{i}}-\mathbf{F}_{\mathbf{0}}^{\mathbf{i}}\right)=0
$$

Equation (2) is a nonlinear function of the nodal coordinates, making it a non-trivial constraint to handle. In the building industry, envelopes are discretised with up to thousands of components, so that the optimisation is done with thousands of constraints.

\subsection{Design-space exploration by structural optimisation}

Figure 1 recalls the three aspects of structural optimisation and the way they will be addressed in the proposed methodology. The geometry is defined with the so-called marionette technique presented in Section 2.3, the mechanical model considers isotropic shell modelled with the finite element technique. The optima are found with derivativefree algorithms. Two families of exploration strategies are envisioned to demonstrate the possibilities offered by Marionette meshes.

The first one considers a unique optimisation criterion and compares the results of optimisation both for the
NURBS and Marionette method. Having a unique criterion makes the comparison of both design strategies more straightforward and some tendencies can be derived from this study.

The second strategy considers several optimisation criteria. In real-life projects, engineers have indeed to deal with different constraints and have often opposing objectives. Solutions that satisfy all optimisation criteria at once do not generally exist, but design belonging to Pareto fronts are the most preferable options in practice. In conceptual design, global optimisation can be preferred to generate possibly unexpected efficient designs.

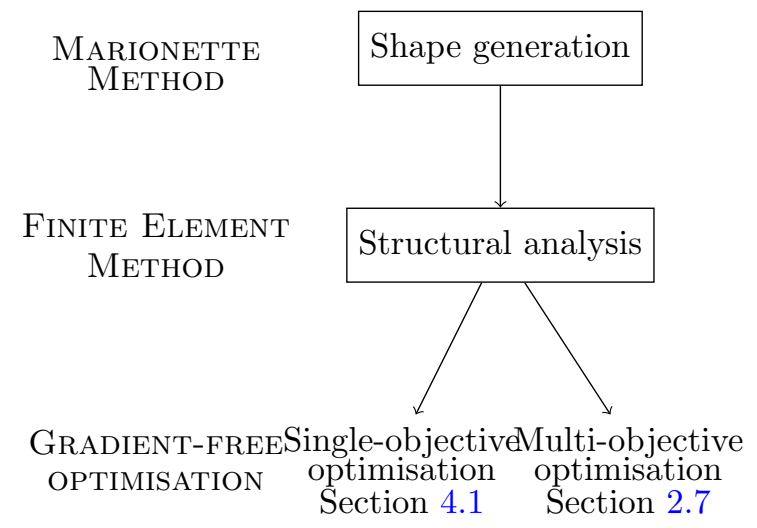

Figure 1: Framework for the shape optimisation of shells

\subsection{The marionette method as a CAD tool}

This article extends the marionette technique [28], which generates exact PQ-Meshes by specifying a plane view and several elevations and by solving a linear system of equations. Indeed, it can be noticed that once the coordinates in the $(x, y)$ plane are chosen, equation (2) becomes a linear equation with respect to the $z$ components of the points $\mathbf{F}_{\mathbf{j}}^{\mathbf{i}}$. Writing $\Delta z_{i}=z_{i}-z_{0}$ and $d_{i j}=\Delta x_{i} \cdot \Delta y_{j}-\Delta x_{j} \cdot \Delta y_{i}$, equation (2) becomes equation (3).

$$
\Delta z_{1} \cdot d_{23}+\Delta z_{2} \cdot d_{31}+\Delta z_{3} \cdot d_{12}=0
$$

For a quadrilateral with a given plane view, there is thus one unique planarity constraint defined by a linear equality. The linear constraints can then be assembled in a matrix form, and an efficient solution is found in [28]: the plane view is determined by the designer, and the altitudes of the nodes are found so that the planarity constraint is satisfied.

Figure 2 illustrates a basic situation handled by the marionette technique: one plane-view and two elevations highlighted in blue and orange in the figure, are sufficient to determine a unique mesh covered with planar quadrilaterals. At the intersection of the elevation curves, the quad has three prescribed altitudes and can thus be 'lifted' according to equation (3): the new point is highlight in black on the left of Figure 2. The lifting method can then 
be applied to two other quads (middle of Figure 2), and propagated iteratively throughout the whole mesh (right of Figure 2). This example is treated in detail in [28], where it is shown that the 'lifting' of the in-plane view is equivalent to the solution of a Cauchy-type problem. The two elevations do not have to be on the boundary of the quadrilateral mesh, in fact, any set of intersecting curves can be used as elevation curves. More complex topologies and patterns can be modelled with the method, as shown in [28]. The marionette technique is a real-time tool because it is based upon the solving of a linear system of equations. For the sake of simplicity, only the vertical component of the nodal coordinates are considered, written $\mathbf{x}$, the planarity constraint can be written as follows:

$$
\mathbf{A} \cdot \mathbf{x}=\mathbf{0}, \mathbf{x} \in \mathbb{R}^{n_{v}}, \mathbf{A} \in \mathcal{M}\left(n_{c}, n_{v}\right)
$$

Where $n_{v}$ is the number of vertices, and $n_{c}$ the number of planarity constraints. When the mesh is constituted of quadrilaterals only, $n_{c}$ is equal to the number of facets. Admissible design with planar facets are found by computing the kernel of $\mathbf{A}$. A basis can be computed, for example with SVD, and writing $\mathbf{N}$ a matrix in $\mathcal{M}\left(n_{v}, d\right)$ that contains vectors of the basis in its columns. A vector $\mathbf{x}_{\mathbf{P}}$ belonging to the kernel of $\mathbf{A}$ can be written as:

$$
\mathbf{x}_{\mathbf{P}}=\mathbf{N} \cdot \widetilde{\mathbf{x}}
$$

The components of the matrix $\mathbf{N}$ depend on the prescribed plane view. With the algorithm proposed in [28] for the solution of the marionette problem, the solution takes typically 5 milliseconds for a mesh with 1000 facets.

In this article, the marionette technique is adapted into a CAD tool similarly to NURBS. It is proposed here to parametrise the plane view by NURBS (or T-splines) and to use Bézier splines or NURBS curves for the elevation curves. The main addition of this article compared to previous implementations of the marionette technique is the possibility to use different degrees and number of control poits for the elevation curves and in-plane view, which allows for a tailor-made parametrisation.

The shape generation is stepwise: first the in-plane view is constructed by meshing the planar NURBS patch along its iso-parametric curves; the $3 \mathrm{D}$ mesh with planar facets is then reconstructed by using the marionette technique according to equation (5). The designer controls thus the plane view and elevation curves. This method is illustrated in Figure 3, where the plane view is controlled by a NURBS patch with nine control points. The two elevations are Bézier splines with four and five control points respectively. Notice that the marionette method naturally decouples the description of in-plane and out-of plane views. The number of control points used for the elevations and the in-plane view do not have to be consistent, like in the example of Figure 3. The number of shape parameters $d_{\text {Marionette }}$ of a pseudo-NURBS marionette mesh for a patch with $N \times M$ control points is thus:

$$
d_{\text {Marionette }}=2 N M+N_{1}+N_{2}-1
$$

where $N_{1}$ and $N_{2}$ are the number of shape parameters for each elevation curve, for example, the number of control points of a Bézier spline. The number of shape parameters $d_{N U R B S}$ offered by NURBS modelling is simply given by:

$$
d_{N U R B S}=3 N M
$$

In our example, $N=M=3, N_{1}=4$ and $N_{2}=5$ : there are thus $18+4+5-1=26$ shape parameters for the marionette mesh, which is close to the number of shape parameters of a NURBS patch with nine control points. Notice however that the two design spaces are different, since discretizations of NURBS do not usually yield planar quadrilaterals. This decoupling of horizontal and vertical description of the shape is a difference with NURBS modelling and can be an opportunity for structural optimisation.

It is noticed that the planarity constraint is satisfied exactly on all the facets. Gridshells or formworks are typically constituted of thousands of faces. The introduction of the marionette technique for CAD-based optimisation modifies the initial formulation of a constrained optimisation into an unconstrained optimisation: using the notations of equations (5) and (4), the optimisation problem reads as:

$$
\min _{\mathbf{A} \mathbf{x}=\mathbf{0}} f(\mathbf{x})=\min _{\widetilde{\mathbf{x}}} f(\mathbf{N} \widetilde{\mathbf{x}})
$$

This high number of constraints and the non-linearity of equation (2) would make the use of optimisation under planarity constraint difficult in practice for other CAD based approaches. The marionette framework guarantees thus a proper parametrisation of a CAD design space with fabrication constraint.

Because the proposed CAD-based approach for marionette mesh relies on NURBS or B-splines, it benefits from some of its advantages, like the possibility to perform degree elevation, through degree elevation of the control mesh and guiding curves. This allows for nested parametrisation of CAD-based Marionette technique, where degree elevation can be performed while preserving a given shape. The technique of nested parameterisation is successfully used in the context of NURBSbased shape optimisation in [29]. In the followings, all the weights of NURBS patches are uniform, so that the surfaces considered in the study are Bézier surfaces. The weight could however be added as shape parameters in the structural optimisation process.

\subsection{Mesh uniformity and re-parametrisation}

Facet planarity is not the only fabrication constraint depending on a surface parametrisation. The aspect ratio of panels should also be controlled in order to ensure constructability of the envelope. The marionette technique as described in [28] can yield meshes subject to undesirable aspect ratios. This is illustrated in Figure 4: a regular plane view yields an irregular mesh, as the discretisation of the elevation curves is not uniform. It is indeed 

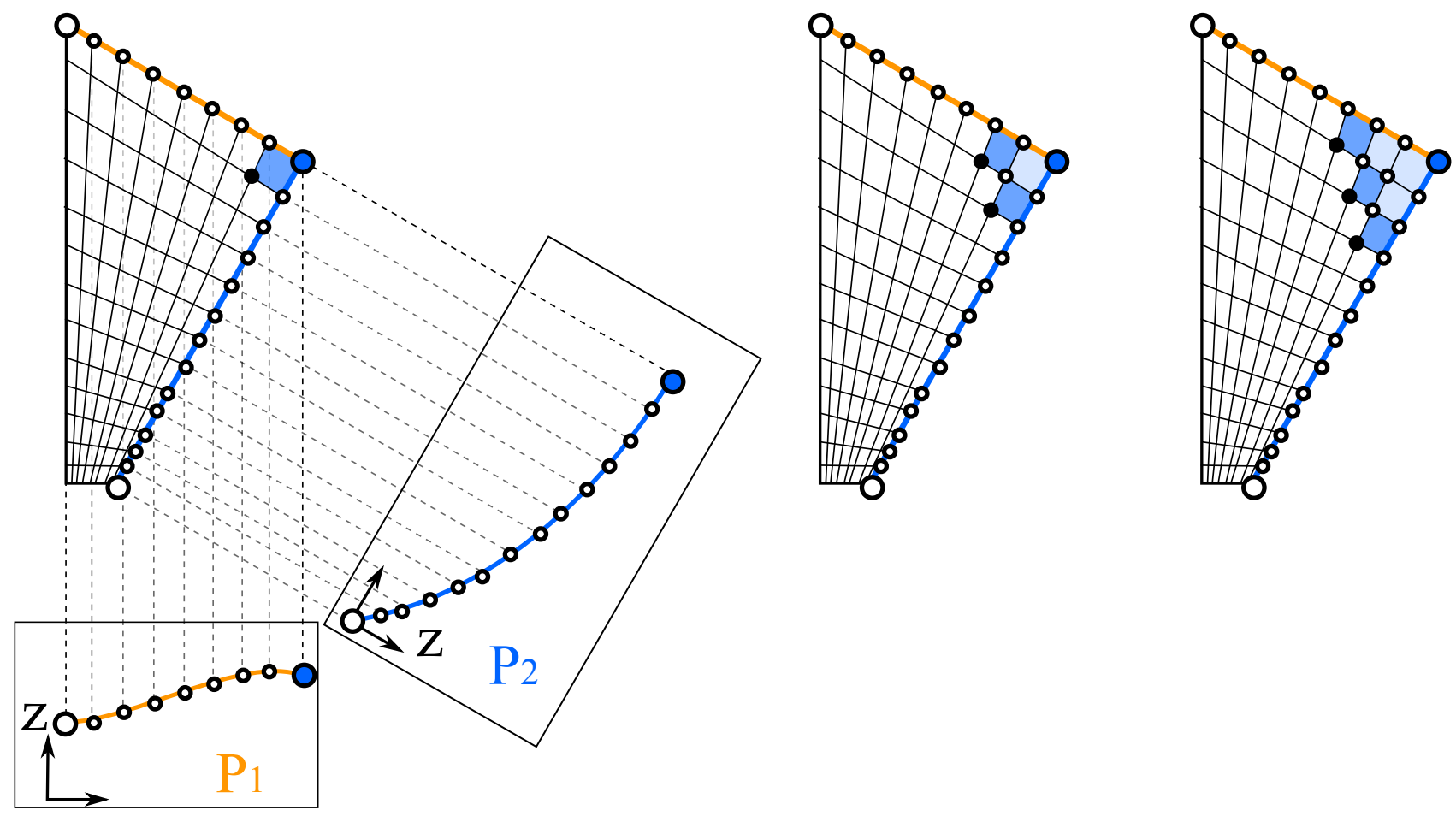

Figure 2: The Marionette method applied to a mesh (from left to right: steps 1,2 \&3): the prescription of two elevation curves allows to iteratively determine a mesh with planar facets.

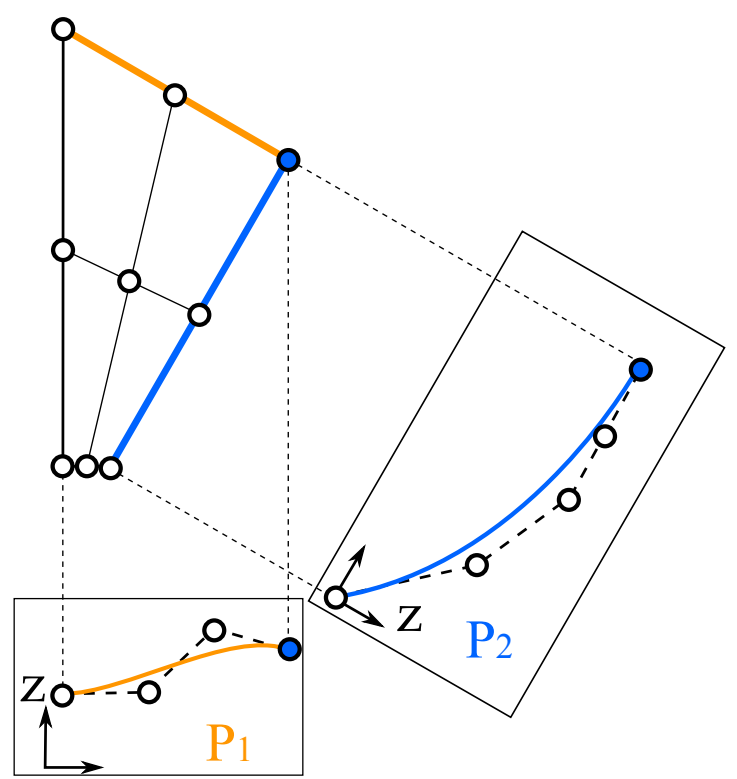

Figure 3: The Marionette method as an alternative to NURBS. easy to notice that the change of length implied by the lift is proportional to $\frac{1}{\cos \alpha}$, where $\alpha$ is the slope of the edge with respect to the horizontal plane. A method is

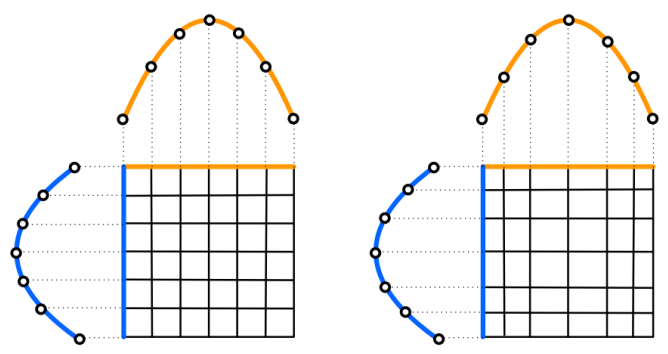

Figure 4: Two parametrisations for a marionette mesh: on the left, a regular plane view yields an irregular mesh, while the plane view on the right yields a mesh with constant length.

proposed here to control the aspect ratio of marionette meshes. The plane view is re-parametrised so that the elevation curves have a constant-length discretisation, as shown on the right of Figure 4. Unless specific cases are considered, the edge-length cannot be a constant on the whole mesh, so that the procedure is applied to all the possible elevation curves. The best suited elevation curve is selected as the minimiser of the variance of lengths on the whole mesh.

\subsection{Mechanical model}

Mechanical assumptions. In the following, shells are modelled as Kirchhoff-Love shells. Indeed, only thin shells, 
where the contribution of shear to the total strain energy can be neglected, are considered. Writing $L$ the span of the structures, the shells have a constant thickness $d$ so that $\frac{d}{L}=\frac{1}{400}$ for the dome and $\frac{d}{L}=\frac{1}{250}$ for the shell on three supports. The shell element used is the TRIC shell element [30]. The number of elements is chosen according to a convergence study. The material is similar to concrete $C 25 / 30$, with a Young's modulus of $E=30 G P a$ and a specific weight of $25 \mathrm{kN} / \mathrm{m}^{3}$.

The number of facets is chosen according to a convergence study shown in Figure 5 and 6, which display strain energy and buckling load factor with respect to the number of elements for the initial configuration of the marionette meshes in section 3. With 6400 triangular elements, the relative errors on strain energy and buckling loads are below $2.5 \%$. The computation time is of $800 \mathrm{~ms}$ on average for linear analysis, while the generation of geometry with planar facets with the marionette technique requires less than $10 \mathrm{~ms}$, with a processor with $2.4 \mathrm{GHz}$ and $2 \mathrm{~Gb}$ memory.

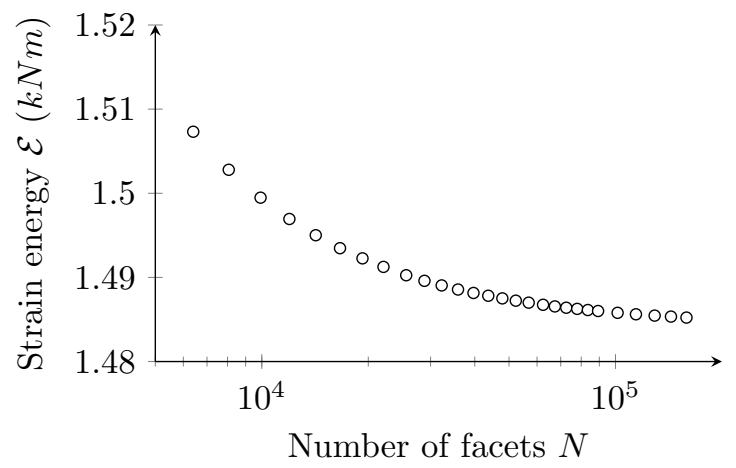

Figure 5: Convergence study on strain energy.

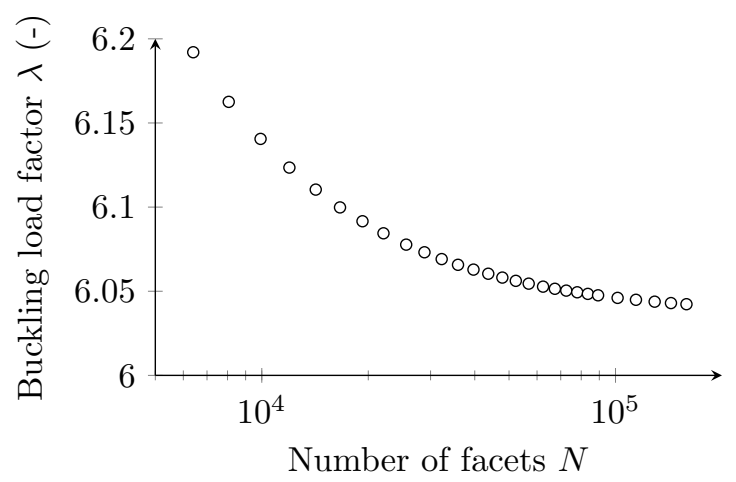

Figure 6: Convergence study on buckling load.

Translations of supports are fully restrained, whereas rotations are allowed. Therefore, the boundary conditions do not satisfy assumptions made in membrane shell theory which restricts translations only in the osculating plane. An immediate consequence is that bending inevitably occurs in the shells. The assumption made for boundary conditions is justified by practical considerations for fabrication of the supports.

Loads. Two load cases are considered in the present study. The first one corresponds to the self-weight of the structure. The second is a non-symmetrical vertical load (pointing downwards) of $1 \mathrm{kPa}$ applied to the coloured sectors of Figure 7. Such load case represents an idealised snow load. In reality, a snow load or a wind load would have a symmetrical component. A non-symmetrical load is chosen to highlight the structural behaviour of the shell under various load conditions.
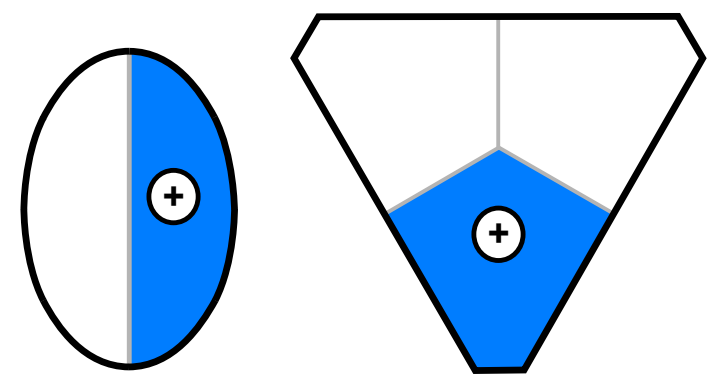

Figure 7: Non-symmetrical load distribution pattern.

\subsection{Implementation of the single-objective optimisation}

This article uses a classical numerical procedure for structural optimisation, where optimisation variables are the design variables only. This approach is the conventional approach in structural optimisation [27]:, it is also called Nested Analysis And Design (NAND) [31].

1. Initialisation of the variables;

2. Generation of the FE model;

3. Calculation of the structural response;

4. Evaluation of the objective function;

5. Computation of the updated parameters after numerical optimisation;

6. Iterate from step 2.

As mentioned in the introduction, the authors explore here the geometrical aspect of structural optimisation. Commercially-available or open-source software for optimisation exist: for the mathematical solution, the NLOpt library [32], which implements gradient-free optimisation, is used. This library was used in structural optimisation by [4], where a comparison of the relative performance of all the algorithms is performed. A discussion on the relative performances of optimisation algorithms for shape optimisation of shell structures is also made in [33].

The result of optimisation depends usually on the initialisation, but also on the numerical scheme used for solving step 5. For this reason, two types of optimisation algorithms are used: a local optimisation algorithm, which is expected to converge towards a local minimum of the 
objective function, and a global algorithm, that is to converge towards the global minimum. Local optimisation algorithms are important in practice, since formal freedom is usually restricted in shape optimisation for construction and one often looks for an optimal design in the vicinity of a sketch provided by the architect and structural engineers. Some global optimisation algorithms, like the one used in this article, converge towards the global minimum. In the present article, global optimisation is used as a reference to find the global minimum of the objective function. The two algorithms used follow:

COBYLA: it is an algorithm for constrained optimisation problems. POWELL defines his algorithm in [34] as follows: 'The COBYLA software [35] constructs linear polynomial approximations to the objective and constraint functions by interpolation at the vertices of simplices (a simplex in $n$ dimensions is the convex hull of $n+1$ points, $n$ being the number of variables).' At each step of the optimisation, a linear programming problem is solved, its result is then evaluated and its value is used to improve the linear approximation.

DIRECT: this algorithm samples the search space and creates hyper-rectangle, based on function evaluations, it identifies potentially optimal hyper-rectangles and decides where to subdivide for the next step [36]. DIRECT is a global algorithm and eventually converges to the global optimum. The number of function calls can however be important, but it remains an efficient algorithm in its category [37].

Optimisation algorithms usually run uninterruptedly if a stopping criterion is not set. Such criterion can be based on a maximal running time or a maximum number of iterations. In the present study, the optimisation time is restricted to 15 minutes for one optimisation run based on elastic linear analysis and 30 minutes for linear buckling analysis with a computer with a standard computer (2.4GHz and $2 \mathrm{~GB}$ memory). An additional convergence criterion on the relative change of the optimum between two optimisation steps is set to 0.001 . The maximal running time considers practical applications: in conceptual design stages, an engineer cannot spend much time on analysis and requires a quick feedback. COBYLA regularly converges under 10 minutes, but global algorithms converge much more slowly because they explore the whole design space.

\subsection{Multi-objective optimisation}

In the followings, for multi-objective optimisation, an evolutionary algorithms using the hypervolume method is used with the HypE reduction and HypE mutation methods that address specific issues linked to the computation of hypervolumes in high dimension, although this article deals mainly with few objectives [38]. The software used is Octopus for GRAsshopper, which implements those algorithms.

In practice, the maximal displacements and the buckling loads are constraints imposed by serviceability limit states (SLS) and ultimate limit states (ULS) requirements, and can be handled with constrained optimisation. Multiobjective optimisation (MOO) is useful when dealing with competing objective (for example minimisation of structural mass vs. acoustics or thermal performance). It is used here as a tool to compare in detail two design spaces by providing a cartography of their respective performances. The population size is set to 200 indiviuals, with 150 generations, which represents 30,000 individual evaluations, which guarantees a good representation of the Pareto front.

\section{Shape optimisation of a dome}

\subsection{Geometric and mechanical constraints}

The first case-study is a shell supported on a closed curve defined as a quadratic Bézier spline. Its topology is represented in Figure 8. It has a width of $L=40$ meters and a length of 57 meters. Because the focus is set here on shape parametrisation, the thickness is not considered as a variable, although it could be easily done, a constant shell thickness $d$ is used, so that $\frac{d}{L}=\frac{1}{400}$. The problem has two axis of symmetry, so that the definition of the geometry can be simplified by focusing on one quarter of the structure (analysis are performed on the whole geometry). The NURBS model is generated with two NURBS patches and the plane view of the marionette mesh with two NURBS patches as well. The elevation curves of the marionette mesh are represented in light orange in the middle of Figure 8. The parametrisation is kept as simple as possible: the two NURBS patches have both $3 \times 3$ control points and they share a common boundary, so that there are 15 control points in total. Uniform weights are used, so that only the position of the control points are used as shape parameters. The NURBS surface is thus a Bézier surface. The notations for the control points are given in Figure 8.
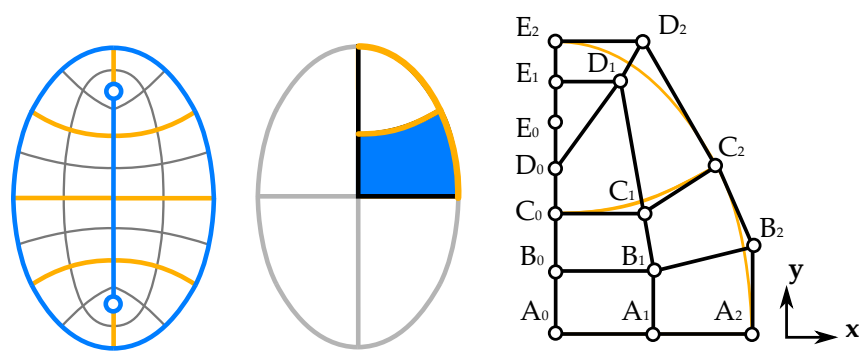

Figure 8: Parametrisation of the elliptic dome

Normal discontinuities are not allowed in the definition of the geometry the target surfaces are $C^{1}$, except for the domains depending on the control points $D_{0} E_{0}$ and $E_{0} E_{1}$, where alignment of the control points from both sides of the patches junction cannot be ensured, so that some constraints on the positions of the control points arise. Table 1 shows the shape parameters for the NURBS and Marionette technique. The geometry of the contour 
is considered as a given constraint. All the points with the subscript 2 are fixed, both in the NURBS and Marionette models, and are not shown in the table. The sign - indicates a constraint enforced by the symmetries of the problem. For example, the points $\mathbf{B}_{\mathbf{1}}, \mathbf{C}_{\mathbf{1}}, \mathbf{D}_{\mathbf{1}}$ have to be aligned in order to guarantee a $C^{1}$ continuity of the surface parametrisation. There are 6 shape parameters in the plane view for both models and two shape parameters on the elevation for the NURBS.

\begin{tabular}{|c|c|c|}
\hline & Marionette & NURBS \\
\hline $\mathbf{A}_{\mathbf{0}}$ & Fixed & $z$ \\
\hline $\mathbf{A}_{\mathbf{1}}$ & $x$ & $x, \mathbf{A}_{\mathbf{0}} \cdot z$ \\
\hline $\mathbf{B}_{\mathbf{0}}$ & $y, z$ & $y, z$ \\
\hline $\mathbf{B}_{\mathbf{1}}$ & $\mathbf{A}_{\mathbf{1}} \cdot x, \mathbf{B}_{\mathbf{0}} \cdot y$ & $\mathbf{A}_{\mathbf{1}} \cdot x, \mathbf{B}_{\mathbf{0}} \cdot y, \mathbf{B}_{\mathbf{0}} \cdot z$ \\
\hline $\mathbf{C}_{\mathbf{0}}$ & $y, \mathbf{B}_{\mathbf{0}} \cdot z$ & $y, z$ \\
\hline $\mathbf{C}_{\mathbf{1}}$ & $x, \mathbf{C}_{\mathbf{0}} \cdot y, \mathbf{C}_{\mathbf{0}} \cdot z$ & $x, \mathbf{C}_{\mathbf{0}} \cdot y, \mathbf{C}_{\mathbf{0}} \cdot z$ \\
\hline $\mathbf{D}_{\mathbf{0}}$ & $y$ & $y$ \\
\hline $\mathbf{D}_{\mathbf{1}}$ & - & - \\
\hline $\mathbf{E}_{\mathbf{0}}$ & $y$ & $y,-$ \\
\hline $\mathbf{E}_{\mathbf{1}}$ & $y$ & $y,-$ \\
\hline
\end{tabular}

Table 1: Shape parameters for the marionette and NURBS model in the dome problem.

The elevation curves of the marionette mesh are represented in orange in Figure 8. One elevation is constrained by the boundary. The other elevation is a curve crossing the boundary. It is represented as a quadratic Bézier spline. The continuity between patches creates one shape parameter. The marionette mesh model has 7 shape parameters, whereas the NURBS model has 8 shape parameters. They are therefore expected to have similar performances.

\subsection{Initialisation}

The two models are initialised with similar geometry and similar performance criteria. The initial value is expected to be efficient: it has a parabolic section with a moderate rise-over span ratio $\left(\frac{h}{L}=25 \%\right)$. Figure 9 shows the initial geometries for the NURBS and marionette meshes. The two shape parameterisation techniques have no reason to yield the same surfaces. In order to have initial geometries that are as close as possible, the initial geometry of the NURBS is set by generating a structured grid based on the initial marionette mesh and by solving a fitting problem [39]. The two initial shapes are visually very close and their mechanical performances are similar within a range of $15 \%$. Note that only the local optimisation algorithm COBYLA is sensitive to the initialisation.

\subsection{Results of single objective optimisation}

The results of single objective optimisation are shown in Figures 10, 12 and 13. Figure 10 illustrates the result of minimisation of the maximal displacement under selfweight. The local optimisation yields a marginally better

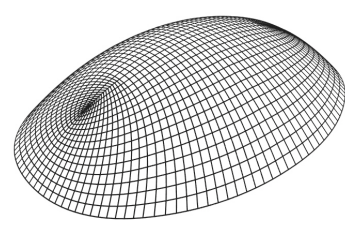

(a) Marionette mesh

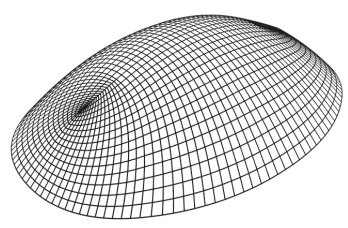

(b) NURBS
Figure 9: Initial geometries for the domes.

result for the marionette design space, but optima of comparable quality are found with the global algorithm.

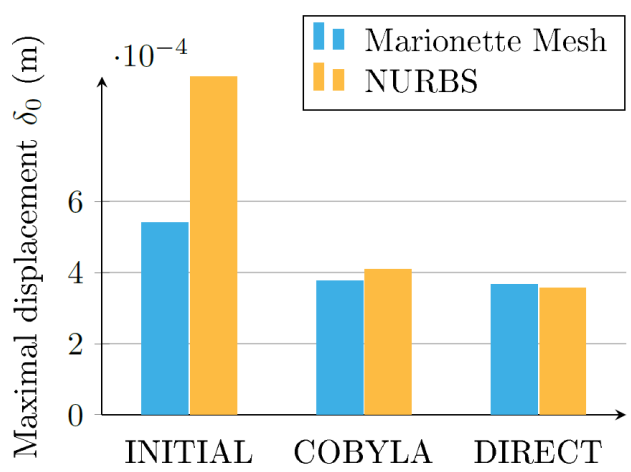

Figure 10: Maximal displacement under self-weight.

The minimisation of strain energy under self-weight shows the same tendency, the difference between the minima of the two design spaces being inferior to $1 \%$.

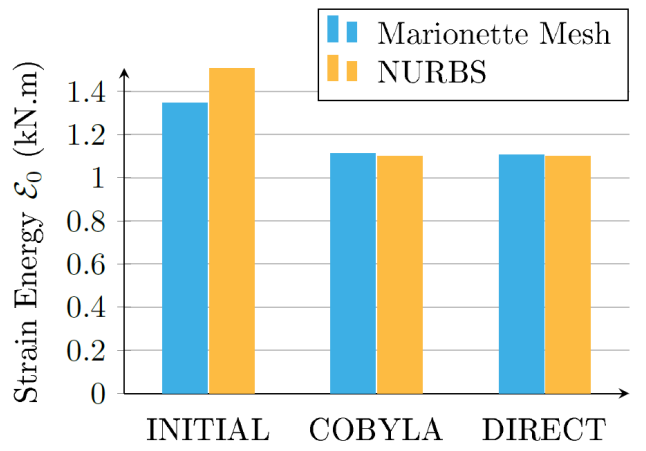

Figure 11: Strain energy under self-weight.

Figure 12 illustrates the result of minimisation of the maximal displacement under non-symmetrical loads. The optima found in the marionette space clearly outperform the ones of the NURBS design space for both local and global optimisation. This tends to highlight a difference between the two design spaces. 


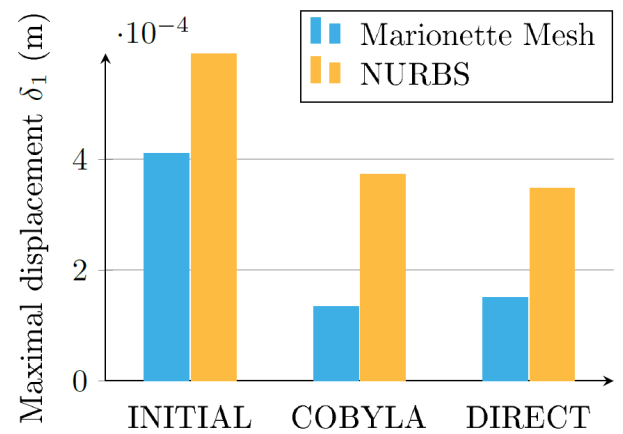

Figure 12: Maximal displacement under non-symmetrical load.

Figure 13 illustrates the result of maximisation of the linear buckling load under self-weight. The optima in both design have similar performance, especially for the global optimisation (DIRECT algorithm).

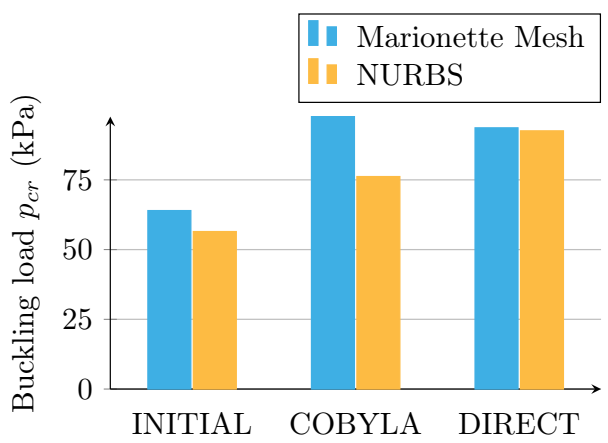

Figure 13: Linear buckling load under self-weight.

The results of local optimisation highlight a better performance of optimisation in the marionette design space. The initialisation has however a better performance in the marionette design space, so that the gap observed in the performances of Marionette and NURBS design spaces can be attributed to this difference of initialisation. Note that the NURBS model does not yield planar facets in general, so that having the same geometry in the NURBS and Marionette design space is usually not possible. Results of global optimisation tend to reduce the differences, except for the non-symmetrical load.

The best optima found for the marionette and NURBS design spaces are shown in Figure 14. The optima of the NURBS and Marionette design spaces are visually similar. The minimisation of displacement under non-symmetrical load leads in both cases to an increase of the rise, the rise-over-span ratio reaching finally $50 \%$. This noticeable change in the geometry might explain the various performances of the different optimisation algorithms. In other optimisations, the rise-over-span ratio remains limited to $25 \%$. The in-plane parametrisation changes between the different optima, although it is not as immediate to see its influence. The most simple parameter to interpret is the position of the umbilical point, which tends to move away from the support after optimisation.

\subsection{Results of multi-objective optimisation}

Single-objective optimisation gives a partial information about the performances of a design. For example, a solution that minimises the strain energy does not necessarily have a high buckling load. This section represent the results of multi-objective optimisation in order to highlight areas of compromise for the two design spaces. Optimisation is studied for following criteria: the minimisation of the maximal displacement and maximisation of linear buckling load. Figure 15 represents the Pareto front found by evolutionary algorithm for the marionette framework together with the optima found by single objective optimisation which have been discussed before. Those optima are close to the Pareto frontier.



Figure 15: Pareto front for the objectives 'maximal displacement' and 'linear buckling load' for the marionette design space.

Figure 16 shows the Pareto front for the same objectives and the NURBS design space. The shape and amplitude of the Pareto front are comparable with the one of the Marionette design space. The optimal values of single-objective optimisation with the NURBS design space are also shown. It can be noticed that those optima are far away from the Pareto front compared to what is observed in Figure 15. This confirms our interpretation stating that 'true' optima of the marionette design space are not necessarily better, but they are easier to reach by single-objective optimisation algorithms in the case of the ellipsoidal dome.

The comparison of the two Pareto fronts is shown in Figure 17. The two fronts are close to each other for low values of $\delta_{0}$ : the NURBS front dominates then the front of the marionette design space (for $p_{c r}$ between $80 k P a$ and $95 \mathrm{kPa}$ ). The front of the marionette design space dominates the front of the NURBS design space for high values of $p_{c r}$. The vertical dashed line represents the maximal buckling load found in the NURBS design space.

The multi-objective optimisation demonstrates that the two design spaces have similar performance, and that a variety of designs can be selected following the structural 


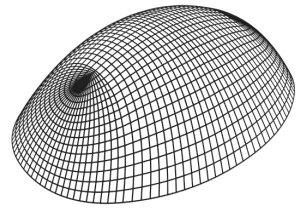

(a) Marionette, optimal $\delta_{0}$

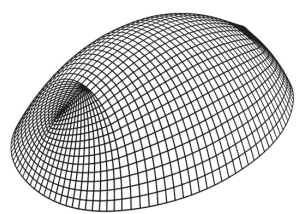

(d) NURBS, optimal $\delta_{0}$



(b) Marionette, optimal $\delta_{1}$

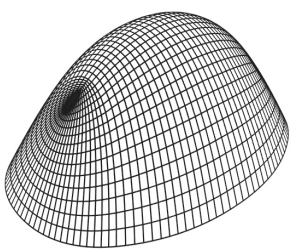

(e) NURBS, optimal $\delta_{1}$

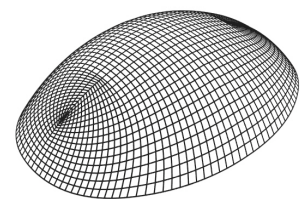

(c) Marionette, optimal buckling

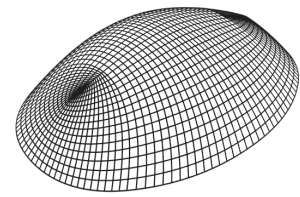

(f) NURBS, optimal buckling

Figure 14: Geometry of the best optima for each objective for the dome. Quads of the marionette design space are all planar, quads of the NURBS design space are not.

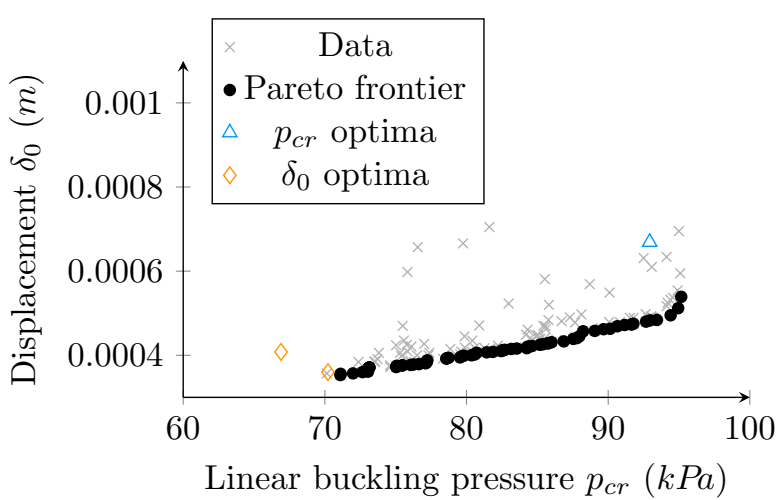

Figure 16: Pareto front for the objectives 'maximal displacement' and 'linear buckling load' for the NURBS design space.

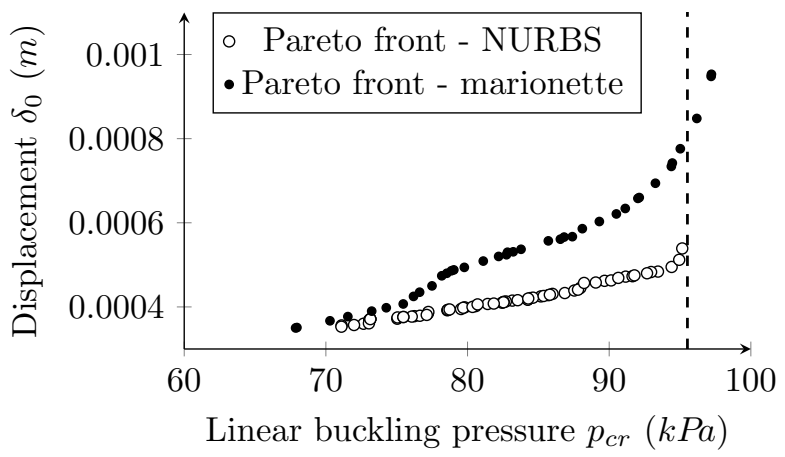

Figure 17: Comparison of the Pareto fronts $\left(p_{c r}, \delta_{0}\right)$ of the two design spaces. imperatives. A second example is studied in the next section in order to confirm these first observations on optimal shell design.

\section{Shape optimisation of a shell on three supports}

\subsection{Geometric and mechanical constraints}

The second case study focuses on a shell supported on three corners. The geometry of the shell is illustrated by Figure 18. The shell has a span arbitrarily set to 130 meters. Its thickness is not a parameter of the optimisation, and is set so that $\frac{t}{L}=\frac{1}{250}$. The geometry has six planes of symmetry and can be decomposed into six domains without singularity, where the marionette technique can be applied. The symmetry is chosen here to simplify the modelling and reduce the number of variables. The NURBS models are built from patches of degree 2 with 9 control points. The plane view of the marionette meshes is built with the same kind of patch, whose control points are shown in Figure 18. The orange area shows the admissible area for the supports of the structure (where $z=0$ ). The shapes are trimmed with a horizontal plane.

Like for the dome, the symmetry and continuity requirements impose constraints on the position of the control points: the NURBS and Marionette patches are $C^{0}$ along the curve $A_{0} C_{0}$ and $C_{0} C_{2}$ on Figure 18. $C^{1}-$ continuity could be enforced by adding additional alignment constraints of points along junction of patches, as already discussed in [1], where continuity patches are introduced to guarantee sufficient continuity. The constraints are recalled in Table 2. NURBS and marionette parametrisations have the same number of shape parameters in the plane, but that the planarity constraint reduces the number of parameters encoding vertical position of control points. 


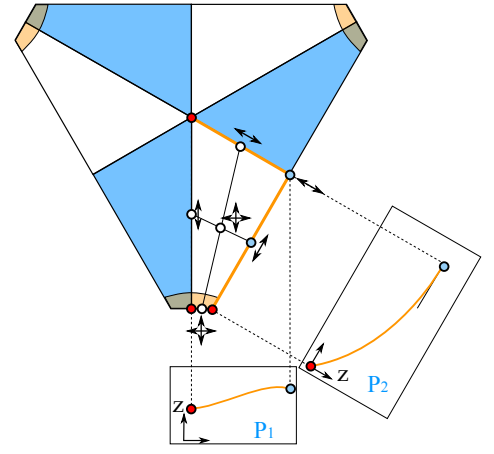

(a) Overall plane view

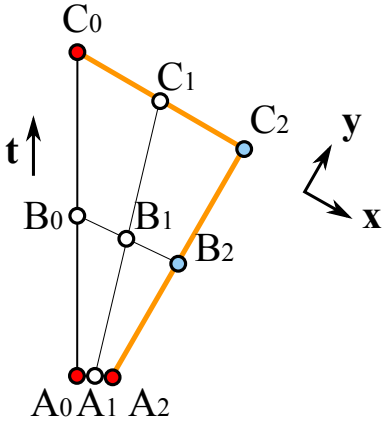

(b) Plane view of one patch

Figure 18: Parametrisation of the CNIT problem with the marionette technique

\begin{tabular}{|c|c|c|}
\hline & Marionette & NURBS \\
\hline $\mathbf{A}_{\mathbf{0}}$ & $t$ & $t$ \\
\hline $\mathbf{A}_{\mathbf{1}}$ & $x, y$ & $x, y$ \\
\hline $\mathbf{A}_{\mathbf{2}}$ & Fixed & Fixed \\
\hline $\mathbf{B}_{\mathbf{0}}$ & $t$ & $t, z$ \\
\hline $\mathbf{B}_{\mathbf{1}}$ & $x, y$ & $x, y, z$ \\
\hline $\mathbf{B}_{\mathbf{2}}$ & $x, y$ & $x, y, z$ \\
\hline $\mathbf{C}_{\mathbf{0}}$ & Fixed & $z$ \\
\hline $\mathbf{C}_{\mathbf{1}}$ & $x$ & $x, z$ \\
\hline $\mathbf{C}_{\mathbf{2}}$ & $\mathbf{C}_{\mathbf{2}} \cdot x=\mathbf{B}_{\mathbf{2}} \cdot x$ & $\mathbf{C}_{\mathbf{2}} \cdot x=\mathbf{B}_{\mathbf{2}} \cdot x, \mathbf{C}_{\mathbf{2}} \cdot z=\mathbf{B}_{\mathbf{2}} \cdot z$ \\
\hline
\end{tabular}

Table 2: Shape parameters for the marionette and NURBS model in the CNIT problem.

The marionette and NURBS models have 9 shape parameters in the horizontal plane respectively. The elevations of the marionette mesh are controlled with quadratic and cubic Bézier splines drawn in Figure 19. In plane $P_{2}$, horizontal tangency at the crown is necessary to preserve $C^{1}$ continuity, which leaves two shape parameters: the height of the crown $h$ and the slope at $\mathbf{A}_{\mathbf{2}}$. There are no restrictions on tangency in plane $P_{1}$, which leaves three shape parameters: the height of three control points. A cubic spline with control points $\left(\mathbf{P}_{\mathbf{i}}\right)$ and parameter $t$ (between 0 and 1 ) is given by following equation:

$\mathbf{B}(t)=(1-t)^{3} \mathbf{P}_{\mathbf{0}}+t \cdot(1-t)^{2} \mathbf{P}_{\mathbf{1}}+t^{2} \cdot(1-t) \mathbf{P}_{\mathbf{2}}+t^{3} \mathbf{P}_{\mathbf{3}}$

For marionette meshes, the $(x, y)$ components are determined by the planar view. Equation (9) has to be modified. The corresponding $u$ value of each raised point on the NURBS patch is retrieved, while a normalised parameter $\bar{u}$ between 0 and 1 is created. The altitudes of the control points are written $z_{i}$, so that for each point to raise $(x(\bar{u}), y(\bar{u}), 0)$, an elevation can be assigned:

$z(\bar{u})=(1-\bar{u})^{3} \cdot z_{0}+\bar{u} \cdot(1-\bar{u})^{2} z_{1}+\bar{u}^{2} \cdot(1-\bar{u}) z_{2}+\bar{u}^{2} \cdot z_{3}$

In total, the marionette mesh and NURBS model both have 16 and 15 parameters respectively. The size of the

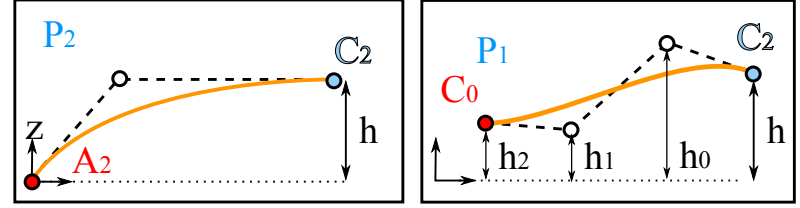

Figure 19: Parametrisation of elevations for the marionette method.

design space of the marionette design space is superior due to enrichment of the description of the elevations for the marionette technique seen in Figure 19.

Supports. Small changes of the shape of the support can radically change the behaviour of a shell. This optimisation parameter is studied in this article by defining an area of possible support location. Some shapes generated with the NURBS or marionette method do not fit within the prescribed domain (delimited by a line). This issue is treated with a penalty method. For a shell with parameter $\mathbf{x}$, we have a constraint on the support $d(\mathbf{x}) \leq 0$. A quadratic penalty is added to the initial objective function to minimise $f(\mathbf{x})$ :

$$
f^{*}(\mathbf{x})=f(\mathbf{x})+\mu g(\mathbf{x})
$$

where

$$
g(\mathbf{x})=\left\{\begin{array}{r}
0 \text { if } d(\mathbf{x}) \leq 0 \\
d(\mathbf{x})^{2} \text { if } d(\mathbf{x})>0
\end{array}\right.
$$

The scalar $\mu$ is a penalty factor, which is set arbitrarily to 1000 . The initialisation is a feasible design, and it was verified that all the algorithms converged indeed to feasible designs.

\subsection{Initialisation}

Like in the previous section, the initial designs are chosen so that they have similar performances in the NURBS and Marionette parametrisations. The two initialisations are shown in Figure 20. The initialisation is in both cases a feasible design in the sense that $g(\mathbf{x})=0$ in (12). There is no curvature at the free-edges, so that local optimisation algorithm can either converge towards negatively or positively curved edge. The structures shown in Figure 20 have a low gaussian curvature: structural optimisation should lead to an increase of curvature and significantly improve the efficiency of the initial design.

\subsection{Results of single objective optimisation}

The results of minimisation of displacement are shown in Figure 21 and 23. The assumption $\frac{t}{L}=\frac{1}{250}$ corresponds to a thickness of 52 centimeters, which corresponds to a self-weight of $13 k P a$. The load applied for the self-weight load case in thus much bigger than the non-symmetrical load. Relative values of displacement $\delta_{0}$ and $\delta_{1}$ should thus be considered with this remark in mind. Notice that this issue arises for large-span structures, where the selfweight is not negligible. Beyond some critical span, single 


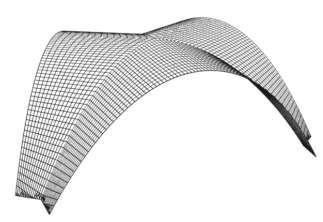

(a) Marionette mesh

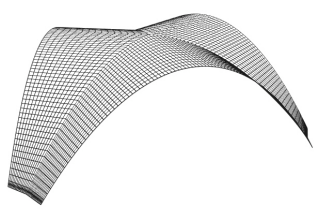

(b) NURBS
Figure 20: Initial geometries for the shells on three supports.

layer thin shells require too much material and other more labour intensive structural systems should be used, like ribbed shells or double-layer shells.

It appears that for all the objective functions, the optimisation drastically improves the performance of the shells compared to the initial design: the maximal displacement under self-weight are reduced by $89 \%$ for the marionette design space and $82 \%$ for the NURBS design space. The optimal values for displacement in the marionette and NURBS design space are similar within a range of $10 \%$.

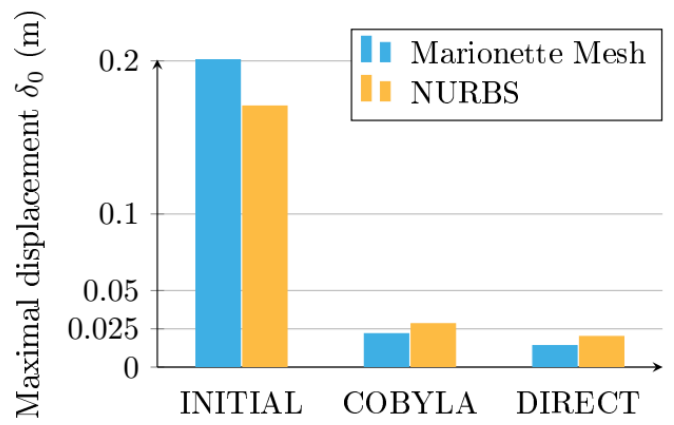

Figure 21: Maximal displacement under self-weight.

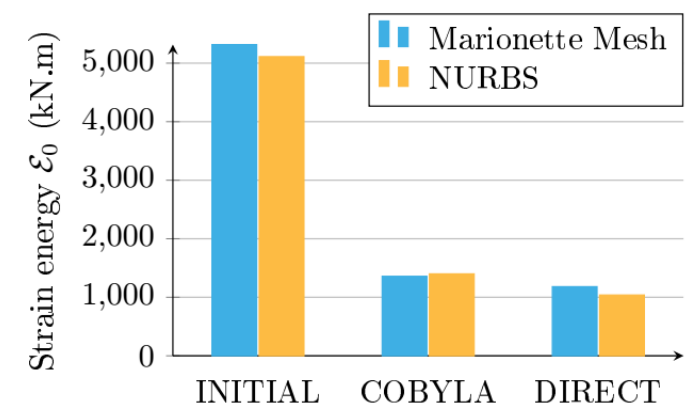

Figure 22: Strain energy under self-weight.

The minimisation of strain energy yields similar results: the reduction of strain energy is $74 \%$ and $72 \%$ for the marionette and NURBS design space respectively. This is comparable to the reduction observed by Marino et al. for the optimisation of shells on three supports based on the geometry of the Kresge auditorium at MIT [27].

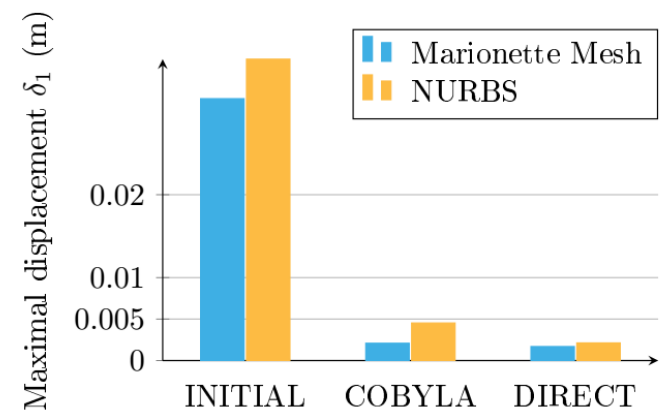

Figure 23: Maximal displacement under non-symmetrical load.

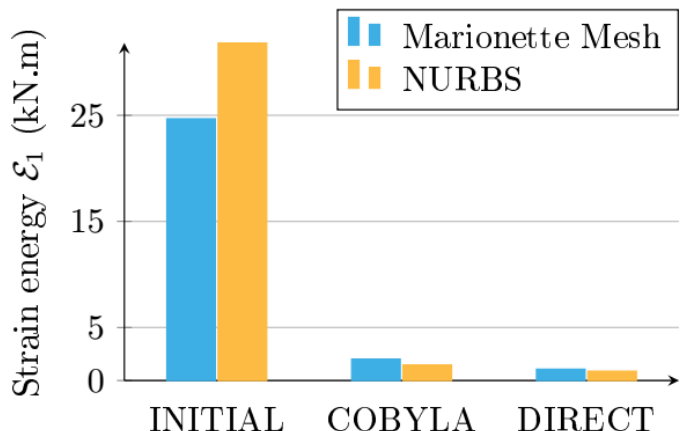

Figure 24: Strain energy under non-symmetrical load.

The maximisation of the buckling capacity highlights a better performance of the optimisation algorithm in the marionette design space, where the relative performance is indeed superior by $50 \%$. This difference can be explained with the visualisation of the optimal designs.



Figure 25: Linear buckling load under self-weight.

The best optimal results of the Marionette and NURBS design spaces are shown in Figure 26. This overview of optimal design illustrates the variety of strategies that can be used by designers to improve the performance of shell structures. The solutions are indeed more diverse than the optimal designs of the dome problem in Figure 14. Optimisation algorithms explore different areas of the design space, so that several families of optimal design can 


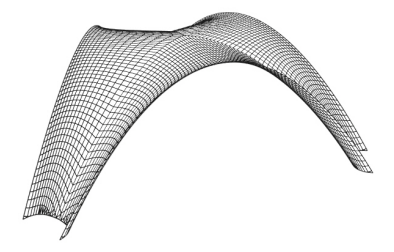

(a) Marionette mesh, optimal $\delta_{0}$

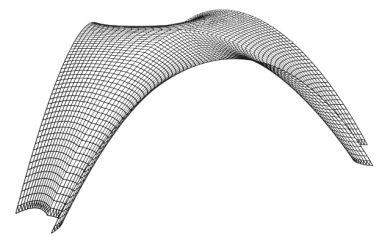

(d) NURBS, optimal $\delta_{0}$

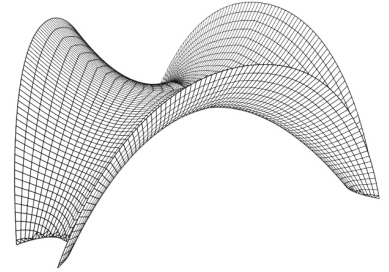

(b) Marionette mesh, optimal $\delta_{1}$

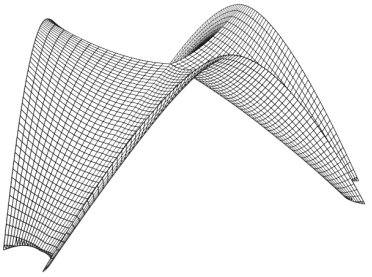

(e) NURBS, optimal $\delta_{1}$

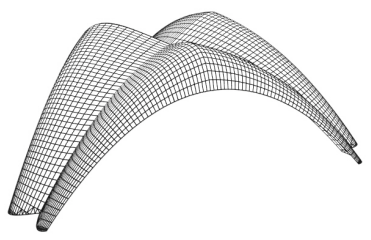

(c) Marionette mesh, optimal buckling

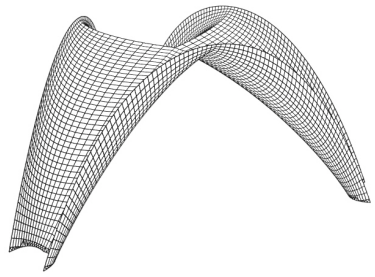

(f) NURBS, optimal buckling

Figure 26: Geometry of the best optima for each objective. Quads of the marionette design space are all planar, quads of the NURBS design space are not.


Figure 27: Displacement pattern under self-weigth in three structures obtained with NURBS patches, seen in plane view: the initial geometry (left), the optimum for displacements (middle) and optimum for strain energy (right).

be identified. The optima of the NURBS and marionette design space have a similar strategy for the minimisation of displacement under self-weight: the rise over-span remains approximately equal to $40 \%$, and negative curvature is introduced locally at the edge, in the manner of some designs by Heinz IsLER. The optimal designs for the minimisation of displacement under non-symmetrical load introduce negative curvature on the whole surface. This solution recalls some shells designed by Felix CANDELA. The shells maximising buckling have a very different strategy: the marionette mesh introduces a crease and the free-edge has a positive gaussian cuvature, whereas the NURBS model has a very high negative curvature at the free-edge.

The displacement pattern of some structures is shown in Figure 27. The displacements are localised in the center of the initial shape, while displacements in optimised structures tend to be more evenly distributed.

\subsection{Results of multi-objective optimisation}

The Pareto fronts found by multi-objective optimisation for the marionette and NURBS design space are repre- sented in Figure 28 and 29 respectively. In both parametrisations, two distinct clusters of optima appear and are highlighted in the Figures.

In the marionette design space, the two clusters highlighted in Figure 28 are non distinguishable in the $\delta_{0} / p_{c r}$ map, but have distinct values of $\delta_{1}$. If one considers the maps $\delta_{0} / p_{c r}$ only, the white cluster dominates the black one. In the two other maps, the black cluster largely dominates the white cluster. This illustrate the interest of considering various load cases.

In Figure 29, the cluster highlighted with white dots features results with low displacements for both load cases, but with low buckling load compared to the cluster in black. This illustrates the importance of choosing the appropriate design criterion for a thin-walled structure. Structures optimised with respect to linear elasticity might have poor performance when non-linearities are considered. Furthermore, our study only considered linear buckling without geometrical imperfections and did not take creep of concrete into account. The bearing capacity of the considered shells is in fact much lower than the values of $p_{c r}$ displayed in the graphs. 


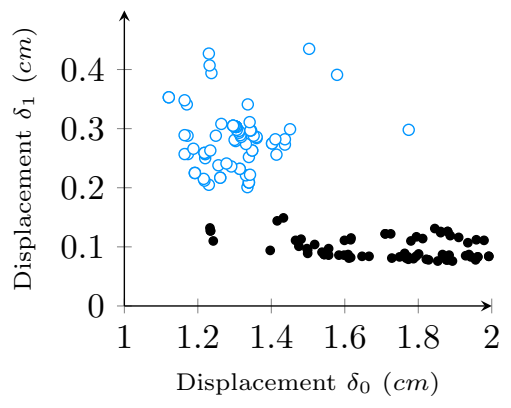

(a) $\delta_{0} / \delta_{1} \operatorname{map}$

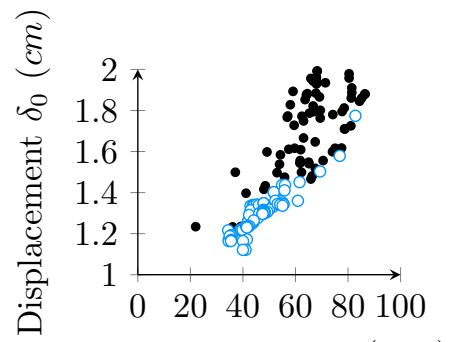

Buckling load $p_{c r}(k P a)$

(b) $p_{c r} / \delta_{0} \operatorname{map}$

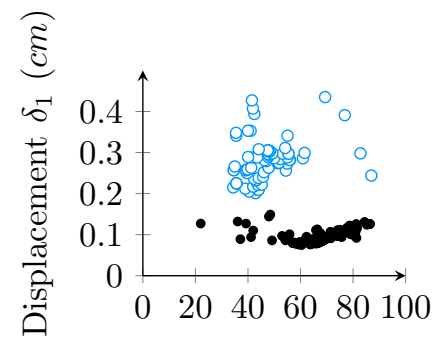

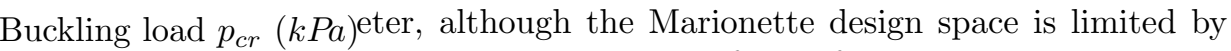

(c) $p_{c r} / \delta_{1}$ map

Figure 28: Pareto front for three objectives for the marionette design space. Two clusters, in two different colours can be identified.

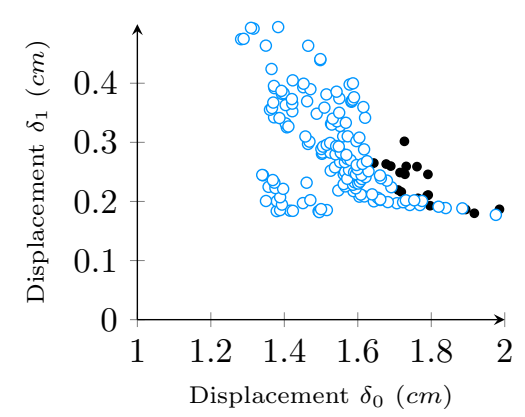

(a) $\delta_{0} / \delta_{1} \operatorname{map}$

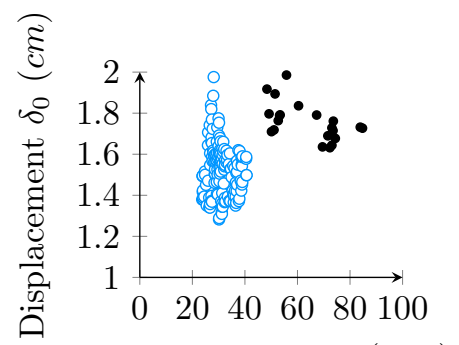

Buckling load $p_{c r}(k P a)$

(b) $p_{c r} / \delta_{0} \operatorname{map}$
Buckling load $p_{c r}(k P a)$

(c) $p_{c r} / \delta_{1} \operatorname{map}$

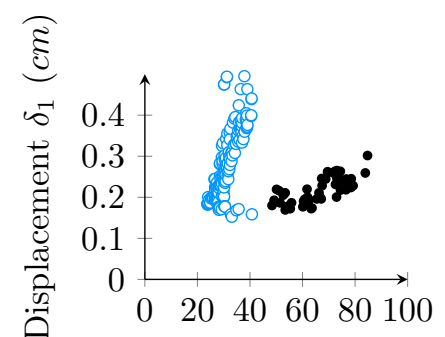

Figure 29: Pareto front for three objectives for the NURBS design space. Two clusters, in two different colours can be identified.

\section{Discussion and guidelines}

\subsection{Performance of the method}

The marionette method provides a rich design space for fabrication-aware shape generation. The performance of optimisation algorithms is comparable to the one of NURBS in the classical examples treated in this article, and when single objective functions are considered, the Marionette method occasionally outperforms NURBS in the present study.

This might seem counter-intuitive, as NURBS offer a wider design space and should feature better global optima. However, the NURBS design space also has many undesirable configurations. Meaningful values for engineers, like rise-over-span ratio or the crease of free-edges are controlled with several parameters with NURBS, instead of one with Marionette meshes.

\subsection{Size of the design spaces}

In our case-study, the size of the Marionette and the planarity constraint for its facets, as seen in equation (6).

It appears that the refinements of the control grid has onette design spaces. Consider equation (6) with $N_{0}=N$ and $N_{1}=M$, the ratio of the sizes of the design spaces $\frac{n_{\text {Mar }}}{n_{N U R B S}}$ is given by equation (13).

$$
\frac{n_{M a r}}{n_{N U R B S}}=\frac{2 N M+N+M-1}{3 N M}
$$

The values of this ratio are plotted in Figure 30. Lower values of $N$ and $M$ yield higher values of $\frac{n_{M}}{n_{N}}$ and less differences between the marionette and NURBS design space. Likewise, patches with a unidirectional refinement (for example with $N$ remaining small while $M$ increases) show little difference between the size of the marionette and NURBS parametrisations.

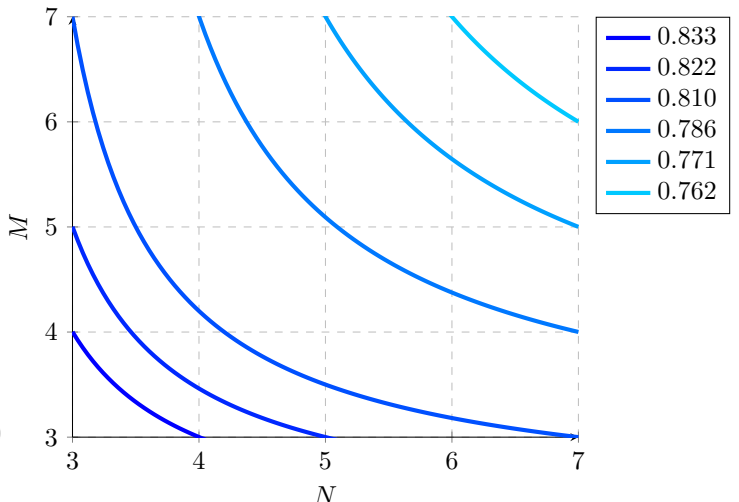

Figure 30: Relative size of the marionette and NURBS design space for one patch of size $N \times M$ an influence of the relative sizes of the NURBS and Mari- 
The choice of problems with few control points made in this $\operatorname{article}(3 \times 3$ patches $)$ is thus beneficial to the marionette technique. Structural optimisation problems parametrised with a refinement of the control grid might show more diversity, and thus potentially better optima in the NURBS design space. Although refinement techniques can be used with the CAD-based marionette technique, the benefits are therefore expected to be of lesser importance than with NURBS-based structural optimisation. It should however be recalled that CAD-based methods are precisely used in order to simplify the optimisation procedure. Geometries parametrised with relatively few control points are therefore more likely to be used in practice.

\subsection{Extensions}

This paper focuses on applications to shell design, but it should be noted that homogenisation approaches can be used to approximate the behaviour of steel gridshells in conceptual design stages. For example, [17] used equivalent orthotropic shell element to find the optimal layout for a quadrangular gridshell. Some patterns, like the kagome grid pattern are isotropic and thus better described with isotropic shell element used in this paper. In [18], it is shown that kagome gridshells, composed of triangles and planar hexagons can be constructed from a PQ-mesh. The results of the present paper could thus be used for the conceptual design of kagome gridshells, which have an isotropic behaviour.

Additionally, smoothness requirements could be added in our optimisation scheme. For example, it is possible to optimise a smoothness metric of a shell under compliance constraint, in the manner of [40], who proposed combined mechanical and geometrical optimisation for shells and ribbed structures.

\subsection{Local explorations}

It appears that some features, like the curvature of freeedges affect drastically the structural behaviour of shell structures. By aligning the mesh with the free-edge, it is possible to control this value with only one parameter, and eases the local exploration of the design space. Moreover, free edges are often visible and the alignment of the panels layout with it can be considered more æsthetically pleasing.

An example of extension is the creation of corrugated shells, much like the CNIT. In NURBS modelling, normal discontinuity would imply manipulation of the knot vector, which could be tedious to parametrise in practice. The marionette technique can use different basis functions and simplify the generation of corrugated structures. Introducing a crease in one elevation curve results in a global corrugation of the surface. This is due to the intrinsic properties of the marionette technique, which solves a discrete version of a hyperbolic equation [28]: the discontinuities of the boundary is propagated throughout the whole surface. Therefore, by controlling the smoothness of one profile curve only, the engineer or architect can modulate the corrugation of the whole surface.

To highlight this point, the elevation curve of the shells on three supports is modified into a non-smooth profil. Equation 10 can be enriched as follows:

$$
z^{\prime}(\bar{u})=z(\bar{u})+h \cdot|\sin (N \pi \bar{u})|
$$

The plot of the crease function of one elevation added in 14 is plotted in Figure 31.

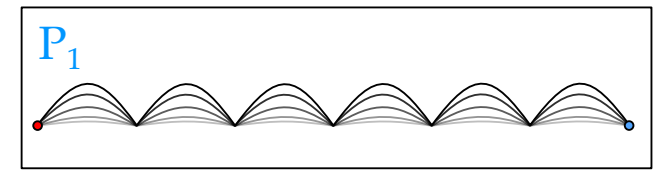

Figure 31: Profile of the crease function, with $(N=6)$

In equation 14, two additional parameters are introduced: the number of undulations $N$ and the crease amplitude $h$. Figure 33 shows the influence of the crease amplitude on the three performance metrics studied in the present paper for a shell on three supports. Some structures are represented in Figure 32. Notice that the whole surfaces are creased although only the elevation curve is modified with our method.

The values are normalised with respect to the noncorrugated shell. The configuration $h=0$ corresponds to the optimum for displacement under symmetrical load found with the COBYLA algorithm. It is noticed that the corrugation leads to an increase of the displacement under symmetrical load and to an improvement of the structural behaviour for the non-symmetrical load and buckling. Corrugation can indeed be interpreted as a mean to increase the bending stiffness of a shell, although the structure transitions from a biaxial state of stress to a uni-axial one [41]. Notice finally that local corrugation significantly increases the critical buckling load of the shell. Our modelling framework allows hence to quantify such statements in a straightforward manner.

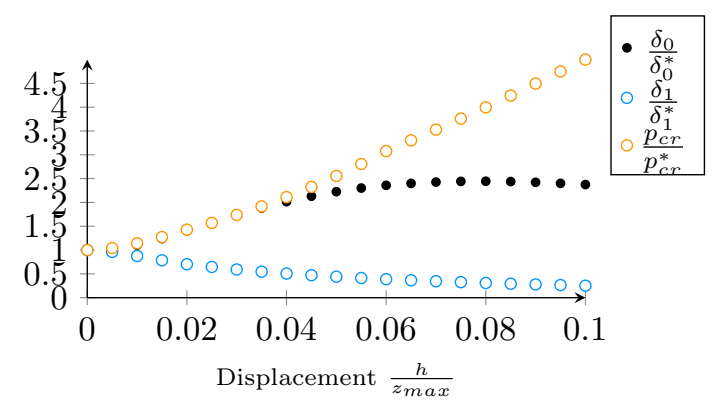

Figure 33: Influence of corrugation amplitude on the performance of a shell

\section{Conclusion}

This paper introduced a geometrical modelling technique for structural optimisation taking into account fab- 

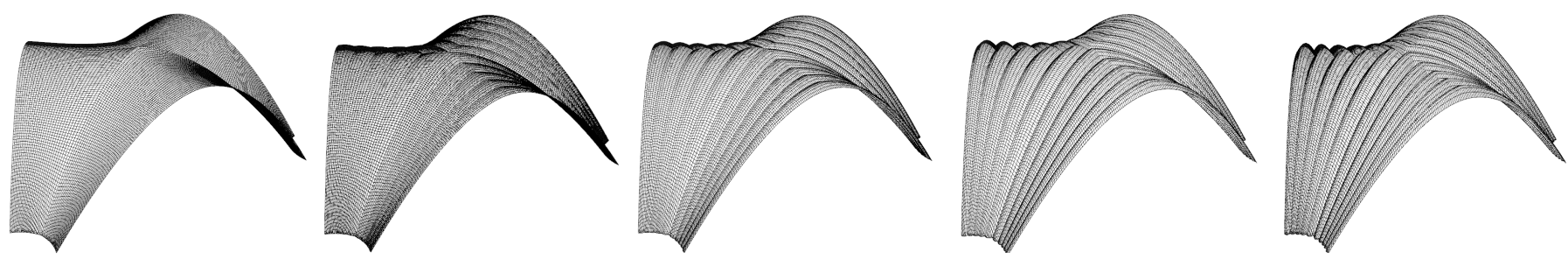

Figure 32: Visualisation of some creased structures (from left to right: $\frac{h}{z_{\max }}=0 ; 0.1 ; 0.25 ; 0.5 ; 0.75$ ). All facets are exactly planar.

rication constraints. The marionette method, which is originally a mesh-based approach, is used here as a CADbased modelling tool. The plane-view and some elevations of the resulting shape can be controlled using NURBS and Bézier splines. The decorrelation between plane-view and elevation allows for the selection of a different number of control points, and thus specific refinement strategies. Such strategies can be used for the geometrical description of free edges, as illustrated in a case-study with a shell on three supports. A comparative study has shown that the proposed framework yields optima similar to the ones found with the ubiquitous NURBS parametrisation. Unlike NURBS modelling, the proposed framework results in meshes with planar quadrilateral facets only.

The authors believe, in the followings of BORGART that fabrication constraints should be integrated within the parametrisation of the search space of structural optimisation algorithms. The constraint of facet planarity is demonstrated to be handled efficiently with the marionette technique. Its potential for the design of glass-covered gridshells seems promising, since the main technological constraint for the envelope is the planarity of panels [42]. Similar work could be conducted for other fabrication constraints, like the proper offset of the support structure in gridshells.

\section{Acknowledgements}

The authors would like to thank the anonymous reviewers and Professor Bernard Maurin for fruitful discussions that lead to a significant improvement of the present paper.

This work was made during Mr. Mesnil doctorate within the framework of an industrial agreement for training through research (CIFRE number 2013/1266) jointly financed by the company Bouygues Construction SA, and the National Association for Research and Technology (ANRT) of France.

[1] K.-U. Bletzinger, E. Ramm, Form finding of shells by structural optimization, Engineering with computers 9 (1) (1993) 27-35.

[2] K. Linkwitz, H. Schek, A new method of analysis of prestressed cable networks and its use on the roofs of the olympic game facilities at munich, in: 9th Congress of IABSE, 1972.

[3] M. Firl, K.-U. Bletzinger, Shape optimization of thin walled structures governed by geometrically nonlinear mechanics, Computer Methods in Applied Mechanics and Engineering.

[4] R. P. Clune, Algorithm selection in structural optimization, Ph.D. thesis, Massachusetts Institute of Technology (2013).
[5] A. Borgart, New challenges for the structural morphology group, Journal of the International Association for Shell and Spatial Structures 51 (3) (2010) 183-189.

[6] J. Glymph, D. Shelden, C. Ceccato, J. Mussel, H. Schober, A parametric strategy for free-form glass structures using quadrilateral planar facets, Automation in Construction 13 (2) (2004) 187-202. doi:10.1016/j.autcon.2003.09.008.

URL http://linkinghub.elsevier.com/retrieve/pii/ S0926580503001055

[7] Y. Liu, W. Wang, H. Pottmann, J. Wallner, Y. Yong-Liang, Geometric Modeling with Conical Meshes and Developable Surfaces, ACM Transactions on Graphics 25 (3) (2006) 681-689.

[8] M. Bagneris, R. Motro, B. Maurin, N. Pauli, Structural Morphology issues in Conceptual Design of Double Curved Systems, International Journal of Space Structures 23 (2) (2008) 79-87.

[9] F. Dischinger, Die rotationsschalen mit unsymmetrischer form und belastung, Bauingenieur 16 (1935) 374.

[10] D. P. Billlington, The Tower and the Bridge: the new art of structural engineering, Princeton University Press, 1985.

[11] J. Schlaich, H. Schober, Glass Roof for the Hippo Zoo at Berlin, Structural Engineering International 7 (4) (1997) 252-254.

[12] R.-q. Feng, J.-m. Ge, Shape optimization method of free-form cable-braced grid shells based on the translational surfaces technique, International Journal of Steel Structures 13 (3) (2013) 435-444.

[13] D. Yang, B. Guo, C. Chong, C. Liu, Modeling and structural behavior of cable-stiffened single-layer latticed domes of hexagonal meshes, Journal of Constructional Steel Research 114 (2015) $237-246$.

[14] R. Mesnil, C. Douthe, O. Baverel, B. Léger, J.-F. Caron, Isogonal moulding surfaces: a family of shapes for high node congruence in free-form structures, Automation in Construction 59 (2015) 38-47.

[15] S. Fujita, M. Ohsaki, Shape optimization of free-form shells using invariants of parametric surface, International Journal of Space Structures 25 (3) (2010) 143-157.

[16] A. Schiftner, J. Balzer, Statics-sensitive layout of planar quadrilateral meshes, Advances in Architectural Geometry 2010 (2010) 221-236.

[17] P. Winslow, S. Pellegrino, S. B. Sharma, Multi-objective optimization of free-form grid structures, Structural and Multidisciplinary Optimization 40 (2010) 257-269. doi:10.1007/ s00158-009-0358-4.

[18] R. Mesnil, C. Douthe, O. Baverel, B. Leger, Linear buckling of quadrangular and kagome gridshells: A comparative assessment, Engineering Structures 132.

[19] K.-U. Bletzinger, M. Firl, J. Linhard, R. Wüchner, Optimal shapes of mechanically motivated surfaces, Computer methods in applied mechanics and engineering 199 (5) (2010) 324-333.

[20] M. Firl, R. Wüchner, K.-U. Bletzinger, Regularization of shape optimization problems using FE-based parametrization, Structural and Multidisciplinary Optimization 47 (4) (2012) 507-521.

[21] E. Stavropoulou, M. Hojjat, K.-U. Bletzinger, In-plane mesh regularization for node-based shape optimization problems, Computer Methods in Applied Mechanics and Engineering 275 (2014) 39-54.

[22] V. Braibant, C. Fleury, Shape optimal design using b-splines, Computer Methods in Applied Mechanics and Engineering 
44 (3) (1984) 247-267.

[23] K.-U. Bletzinger, R. Wüchner, F. Daoud, N. Camprubí, Computational methods for form finding and optimization of shells and membranes, Computer Methods in Applied Mechanics and Engineering 194 (30-33) (2005) 3438-3452.

[24] T. J. R. Hughes, J. A. Cottrell, Y. Bazilevs, Isogeometric analysis: CAD, finite elements, NURBS, exact geometry and mesh refinement, Computer methods in applied mechanics and engineering 194 (39) (2005) 4135-4195.

[25] S.-H. Ha, K. K. Choi, S. Cho, Numerical method for shape optimization using T-spline based isogeometric method, Structural and Multidisciplinary Optimization 42 (3) (2010) 417-428.

[26] E. Ramm, K.-U. Bletzinger, R. Reitinger, Shape optimization of shell structures, Revue Européenne des Éléments Finis 2 (3) (1993) 377-398

[27] E. Marino, L. Salvatori, M. Orlando, C. Borri, Two shape parametrizations for structural optimization of triangular shells, Computers \& Structures 166 (2016) 1-10.

[28] R. Mesnil, O. Baverel, C. Douthe, Marionette meshes: modelling free-form architecture with planar facets, International Journal of Space Structures 32.

[29] J.-A. Désidéri, B. A. El Majd, A. Janka, Nested and selfadaptive bézier parameterizations for shape optimization, Journal of Computational Physics 224 (1) (2007) 117-131. doi: 10.1016/j.jcp.2006.12.016.

[30] J. Argyris, M. Papadrakakis, C. Apostolopoulou, S. Koutsourelakis, The tric shell element: theoretical and numerical investigation, Computer Methods in Applied Mechanics and Engineering 182 (1) (2000) $217-245$. doi:http://dx.doi.org/10. 1016/S0045-7825(99)00094-8.

URL http://www.sciencedirect.com/science/article/pii/ S0045782599000948

[31] J. Arora, Q. Wang, Review of formulations for structural and mechanical system optimization, Structural and Multidisciplinary Optimization 30 (4) (2005) 251-272.

[32] S. G. Johnson, The nlopt nonlinear-optimization package, accessed: 2016-02-11 (2015).

[33] R. Mesnil, Structural exploration of fabrication-aware design spaces for non-standard architecture, Ph.D. thesis, Université Paris-Est (2017).

[34] M. J. Powell, A view of algorithms for optimization without derivatives, Mathematics Today-Bulletin of the Institute of Mathematics and its Applications 43 (5) (2007) 170-174.

[35] M. J. Powell, A direct search optimization method that models the objective and constraint functions by linear interpolation, in: Advances in optimization and numerical analysis, Springer, 1994, pp. 51-67.

[36] D. R. Jones, C. D. Perttunen, B. E. Stuckman, Lipschitzian optimization without the lipschitz constant, Journal of Optimization Theory and Applications 79 (1) (1993) 157-181.

[37] D. E. Finkel, Direct optimization algorithm user guide.

[38] J. Bader, E. Zitzler, Hype: An algorithm for fast hypervolumebased many-objective optimization, Evolutionary computation 19 (1) (2011) 45-76.

[39] W. Ma, J.-P. Kruth, Nurbs curve and surface fitting for reverse engineering, The International Journal of Advanced Manufacturing Technology 14 (12) (1998) 918-927. doi:https: //doi.org/10.1007/BF01179082.

[40] M. Ohsaki, T. Ogawa, R. Tateishi, Shape optimization of curves and surfaces considering fairness metrics and elastic stiffness, Structural and Multidisciplinary Optimization 24 (6) (2002) 449-456.

[41] R. Mesnil, O. Baverel, C. Douthe, J.-F. Caron, B. Léger, Structural morphology and performance of plated structures with planar quadrilateral facets, Journal of the International Association for Shell and Spatial Structures 58 (1) (2017) 7-22.

[42] R. Mesnil, O. Baverel, C. Douthe, Non-standard patterns for gridshell structures: fabrication and structural optimization, Journal of the International Association for Shell and Spatial Structures 58 (4).

\section{Appendix A. Results}

\section{Appendix A.1. Elliptical dome}

The parameter values for the dome structures can be found in Tables A.5 and A.6.

\begin{tabular}{|c|c|}
\hline & Initial value \\
\hline $\mathbf{A}_{\mathbf{0}}$ & $(0,0,-)$ \\
$\mathbf{A}_{\mathbf{1}}$ & $(10.0,0,-)$ \\
$\mathbf{A}_{\mathbf{2}}$ & $(20.0,0,0.0)$ \\
$\mathbf{B}_{\mathbf{0}}$ & $(0,6.69,-)$ \\
$\mathbf{B}_{\mathbf{1}}$ & $(10.38,6.69,-)$ \\
$\mathbf{B}_{\mathbf{2}}$ & $(20.07,11.68,0.0)$ \\
$\mathbf{C}_{\mathbf{0}}$ & $(0.0,13.197,10.0)$ \\
$\mathbf{C}_{\mathbf{1}}$ & $(8.65,13.20,10.0)$ \\
$\mathbf{C}_{\mathbf{2}}$ & $(14.40,20.53,0.0)$ \\
$\mathbf{D}_{\mathbf{0}}$ & $(0.0,16.45,0.0)$ \\
$\mathbf{D}_{\mathbf{1}}$ & $(6.49,22.01,-)$ \\
$\mathbf{D}_{\mathbf{2}}$ & $(9.34,28.42,0.0)$ \\
$\mathbf{E}_{\mathbf{0}}$ & $(0.0,18.753,-)$ \\
$\mathbf{E}_{\mathbf{1}}$ & $(0.0,22.01,-)$ \\
$\mathbf{E}_{\mathbf{2}}$ & $(0.0,28.42,0.0)$ \\
\hline
\end{tabular}

Table A.3: Initial nodal coordinates for the marionette design space (values are expressed in meters).

\begin{tabular}{|c|c|}
\hline & Initial value \\
\hline $\mathbf{A}_{\mathbf{0}}$ & $(0,0,12.8)$ \\
$\mathbf{A}_{\mathbf{1}}$ & $(10.0,0,12.8)$ \\
$\mathbf{A}_{\mathbf{2}}$ & $(20.0,0,0.0)$ \\
$\mathbf{B}_{\mathbf{0}}$ & $(0,6.69,12.8)$ \\
$\mathbf{B}_{\mathbf{1}}$ & $(10.38,6.69,12.8)$ \\
$\mathbf{B}_{\mathbf{2}}$ & $(20.07,11.68,0.0)$ \\
$\mathbf{C}_{\mathbf{0}}$ & $(0.0,13.197,10.0)$ \\
$\mathbf{C}_{\mathbf{1}}$ & $(8.65,13.20,10.0)$ \\
$\mathbf{C}_{\mathbf{2}}$ & $(14.40,20.53,0.0)$ \\
$\mathbf{D}_{\mathbf{0}}$ & $(0.0,16.44,8.60)$ \\
$\mathbf{D}_{\mathbf{1}}$ & $(6.31,22.01,6.21)$ \\
$\mathbf{D}_{\mathbf{2}}$ & $(9.34,28.42,0.0)$ \\
$\mathbf{E}_{\mathbf{0}}$ & $(0.0,18.75,7.61)$ \\
$\mathbf{E}_{\mathbf{1}}$ & $(0.0,22.01,6.21)$ \\
$\mathbf{E}_{\mathbf{2}}$ & $(0.0,28.42,0.0)$ \\
\hline
\end{tabular}

Table A.4: Initial nodal coordinates for the NURBS design space (values are expressed in meters).

\section{Appendix A.2. Shell on three supports}

The parameter values corresponding to the optima found in Section 4.3 are presented in Table A.9. The values preceded by a $\Delta$ indicate a variation from the initial position: there initial value is thus zero. Initial nodal coordinates, in th $(u, t)$ coordinate system are given.

Initial nodal coordinates for the NURBS design space are given in Table A.8. 


\begin{tabular}{|c|c|c|c|c|c|c|c|c|c|c|c|}
\hline & Initial & \multicolumn{2}{|c|}{ Optimal $\delta_{0}$} & \multicolumn{2}{c|}{ Optimal $\mathcal{E}_{0}$} & \multicolumn{2}{c|}{ Optimal $p_{c r}$} & \multicolumn{3}{c|}{ Optimal $\delta_{1}$} \\
& & COBYLA & DIRECT & COBYLA & DIRECT & COBYLA & DIRECT & COBYLA & DIRECT & COBYLA & DIRECT \\
\hline $\boldsymbol{\Delta} \mathbf{A}_{\mathbf{1}} \cdot \mathbf{Y}$ & 0 & -0.1 & 0 & 1.5 & 1.9 & 1.4 & 3 & -2.7 & -1 & -3 & -3 \\
\hline $\boldsymbol{\Delta} \mathbf{B}_{\mathbf{0}} \cdot \mathbf{Y}$ & 0 & 0 & -1.7 & 0.5 & -1 & 0.2 & -1 & 0.3 & 0.9 & 0 & 0.9 \\
\hline $\boldsymbol{\Delta} \mathbf{C}_{\mathbf{0}} \cdot \mathbf{Y}$ & 0 & 1.4 & 0 & 1 & 1.7 & 1.6 & 0 & 1.3 & 1.8 & 2.3 & 2.9 \\
\hline $\boldsymbol{\Delta} \mathbf{C}_{\mathbf{1}} \cdot \mathbf{X}$ & 0 & 0 & 0 & 1.2 & 0.9 & 2.1 & 3 & -1.2 & 0 & -0.8 & -0.4 \\
\hline $\boldsymbol{\Delta} \mathbf{E}_{\mathbf{0}} \cdot \mathbf{Y}$ & 0 & 0.5 & -2.7 & -0.1 & -2.1 & 2 & 3.6 & 3 & 3.9 & 2.2 & 3.9 \\
\hline $\boldsymbol{\Delta} \mathbf{E}_{\mathbf{1}} \cdot \mathbf{Y}$ & 0 & 0 & 1.3 & 1.3 & 2 & 1.4 & 1.2 & 1.2 & 1.9 & 1.1 & 2 \\
\hline$h$ & 10 & 14.81 & 15.65 & 13.38 & 13.06 & 10.58 & 12.5 & 20 & 19.91 & 20 & 19.91 \\
\hline
\end{tabular}

Table A.5: Parameter values for dome in the marionette design space, bold numbers indicate parameters that can be changed by optimisation (values are expressed in meters).

\begin{tabular}{|c|c|c|c|c|c|c|c|c|c|c|c|}
\hline & \multirow[t]{2}{*}{ Initial } & \multicolumn{2}{|c|}{ Optimal $\delta_{0}$} & \multicolumn{2}{|c|}{ Optimal $\mathcal{E}_{0}$} & \multicolumn{2}{|c|}{ Optimal $p_{c r}$} & \multicolumn{2}{|c|}{ Optimal $\delta_{1}$} & \multicolumn{2}{|c|}{ Optimal $\mathcal{E}_{1}$} \\
\hline & & COBYLA & DIRECT & COBYLA & DIRECT & COBYLA & DIRECT & COBYLA & DIRECT & COBYLA & DIRECT \\
\hline$\Delta \mathrm{A}_{1} \cdot \mathrm{Y}$ & 0 & 0.6 & 0.7 & 1.9 & 2 & 0.7 & 2.9 & -1 & -1.7 & 0.4 & 0.3 \\
\hline$\Delta \mathrm{B}_{0} \cdot \mathrm{Y}$ & 0 & 0 & -1.6 & 1.4 & 1.7 & -1.7 & -1 & 0.1 & -0.3 & -0.6 & 0.1 \\
\hline$\Delta C_{0} \cdot Y$ & 0 & 0.9 & -0.9 & 2.5 & 2.2 & -0.4 & 0.7 & 1.9 & -1.7 & -0.1 & -1 \\
\hline$\Delta \mathrm{C}_{1} \cdot \mathrm{X}$ & 0 & -0.5 & -0.4 & 0.8 & 0.7 & 0.6 & 0 & 0.1 & -2.4 & 0.6 & 0 \\
\hline$\Delta \mathrm{E}_{0} \cdot \mathrm{Y}$ & 0 & -1 & -3 & -2.3 & -2.6 & -0.9 & 4 & -1.1 & -3.9 & -0.9 & -4 \\
\hline$\Delta E_{1} \cdot Y$ & 0 & 0.5 & -1.9 & 1.4 & 0.6 & 0.2 & 1.9 & -0.9 & -1.9 & -0.7 & -1.8 \\
\hline$h_{1}$ & 10 & 12.9 & 17.5 & 16.5 & 16.9 & 12.6 & 15 & 13.5 & 15.2 & 13.1 & 15.2 \\
\hline$h_{2}$ & 10 & 9.8 & 14 & 12.7 & 13.3 & 9.8 & 12.7 & 10 & 13.6 & 10.2 & 12.8 \\
\hline
\end{tabular}

Table A.6: Parameter values for dome in the nurbs design space, bold numbers indicate parameters that can be changed by optimisation (values are expressed in meters).

\begin{tabular}{|l|c|}
\hline & Initial value \\
\hline $\mathbf{A}_{\mathbf{0}}$ & $(0,0,-)$ \\
$\mathbf{A}_{\mathbf{1}}$ & $(4.5,0,-)$ \\
$\mathbf{A}_{\mathbf{2}}$ & $(9,0,-)$ \\
$\mathbf{B}_{\mathbf{0}}$ & $(0,42.5,-)$ \\
$\mathbf{B}_{\mathbf{1}}$ & $(13.14,36.21,-)$ \\
$\mathbf{B}_{\mathbf{2}}$ & $(26.28,29.93,-)$ \\
$\mathbf{C}_{\mathbf{0}}$ & $(0.0,85.0,-)$ \\
$\mathbf{C}_{\mathbf{1}}$ & $(21.78,72.43,-)$ \\
$\mathbf{C}_{\mathbf{2}}$ & $(43.56,59.85,-)$ \\
\hline
\end{tabular}

Table A.7: Initial nodal coordinates for the marionette design space (values are expressed in meters).

\begin{tabular}{|c|c|}
\hline & Initial value \\
\hline $\mathbf{A}_{\mathbf{0}}$ & $(0,0,0)$ \\
$\mathbf{A}_{\mathbf{1}}$ & $(4.5,0,0)$ \\
$\mathbf{A}_{\mathbf{2}}$ & $(9,0,0)$ \\
$\mathbf{B}_{\mathbf{0}}$ & $(0,42.5,55)$ \\
$\mathbf{B}_{\mathbf{1}}$ & $(13.14,36.21,55)$ \\
$\mathbf{B}_{\mathbf{2}}$ & $(26.28,29.93,55)$ \\
$\mathbf{C}_{\mathbf{0}}$ & $(0.0,85.0,55)$ \\
$\mathbf{C}_{\mathbf{1}}$ & $(21.78,72.43,55)$ \\
$\mathbf{C}_{\mathbf{2}}$ & $(43.56,59.85,55)$ \\
\hline
\end{tabular}

Table A.8: Initial nodal coordinates for the marionette design space (values are expressed in meters). 


\begin{tabular}{|c|c|c|c|c|c|c|c|c|c|c|c|}
\hline & Initial & \multicolumn{2}{|c|}{ Optimal $\delta_{0}$} & \multicolumn{2}{c|}{ Optimal $\mathcal{E}_{0}$} & \multicolumn{2}{c|}{ Optimal $p_{c r}$} & \multicolumn{2}{c|}{ Optimal $\delta_{1}$} & \multicolumn{2}{c|}{ Optimal $\mathcal{E}_{1}$} \\
& & COBYLA & DIRECT & COBYLA & DIRECT & COBYLA & DIRECT & COBYLA & DIRECT & COBYLA & DIRECT \\
\hline$\Delta \mathbf{A}_{\mathbf{1}} \cdot \mathbf{U}$ & 0 & 2 & 4 & 2 & 4 & -2 & 2 & 3 & 3 & 2 & 0 \\
\hline$\Delta \mathbf{A}_{\mathbf{1}} \cdot \mathbf{T}$ & 0 & 5 & 10 & 4 & 10 & 4 & -8 & 9 & -6 & 7 & -6 \\
\hline$\Delta \mathbf{B}_{\mathbf{0}} \cdot \mathbf{T}$ & 0 & 8 & 10 & 6 & 10 & -3 & 10 & 2 & 7 & 8 & 7 \\
\hline$\Delta \mathbf{C}_{\mathbf{1}} \cdot \mathbf{X}$ & 0 & 6 & -2 & 7 & -2 & 3 & 9 & 10 & 6 & 9 & -3 \\
\hline$\Delta \mathbf{C}_{\mathbf{2}} \cdot \mathbf{X}$ & 0 & 5 & 3 & -2 & 3 & -6 & -9 & 4 & -9 & 0 & -10 \\
\hline$\Delta \mathbf{B}_{\mathbf{1}} \cdot \mathbf{U}$ & 0.0 & 8 & 1 & 4 & -1 & 9 & -8 & 3 & -8 & 6 & 10 \\
\hline $\mathbf{B}_{\mathbf{1}} \cdot \mathbf{T}$ & 0.0 & 7 & 15 & 6 & 15 & 4 & -9 & 5 & -9 & 11 & -10 \\
\hline $\boldsymbol{B}_{\mathbf{2}} \cdot \mathbf{Y}$ & 0.0 & 12 & 3 & 10 & 3 & 4 & 3 & -14 & 3 & -3 & 3 \\
\hline $\mathbf{H}_{\mathbf{0}}$ & 60.0 & 54.5 & 53.1 & 63.4 & 53.1 & 72.9 & 52.5 & 74.9 & 67.5 & 74.5 & 73.8 \\
\hline $\mathbf{H}_{\mathbf{1}}$ & 60.0 & 59.1 & 61.8 & 54.3 & 61.8 & 41.4 & 67.9 & 71.3 & 73.1 & 69.5 & 69.2 \\
\hline $\mathbf{H}_{\mathbf{2}}$ & 60.0 & 58.8 & 57.5 & 57.1 & 57.5 & 74.9 & 61.4 & 58.5 & 57.5 & 62.5 & 45.7 \\
\hline $\mathbf{H}_{\mathbf{3}}$ & 60.0 & 60.0 & 56.9 & 58.8 & 56.9 & 58.5 & 50.0 & 57 & 50.0 & 54.2 & 43.3 \\
\hline $\mathbf{l}$ & 30.26 & 28.16 & 32.09 & 31.80 & 32.09 & 46.62 & 35.57 & 57.5 & 35.57 & 40.83 & 32.10 \\
\hline
\end{tabular}

Table A.9: Parameter values for shell on three supports in the marionette design space (values are expressed in meters).

\begin{tabular}{|c|c|c|c|c|c|c|c|c|c|c|c|}
\hline & Initial & \multicolumn{2}{|c|}{ Optimal $\delta_{0}$} & \multicolumn{2}{c|}{ Optimal $\mathcal{E}_{0}$} & \multicolumn{2}{c|}{ Optimal $p_{c r}$} & \multicolumn{2}{c|}{ Optimal $\delta_{1}$} & \multicolumn{2}{c|}{ Optimal $\mathcal{E}_{1}$} \\
& & COBYLA & DIRECT & COBYLA & DIRECT & COBYLA & DIRECT & COBYLA & DIRECT & COBYLA & DIRECT \\
\hline$\Delta \mathbf{A}_{\mathbf{0}} \cdot \mathbf{T}$ & 1 & 2 & 5 & 4 & 6 & 6 & 4 & 3 & 5 & 6 & 6 \\
\hline$\Delta \mathbf{A}_{\mathbf{1}} \cdot \mathbf{U}$ & 0 & 0 & 3 & 2 & 4 & 0 & 1 & 0 & 0 & 1 & 1 \\
\hline$\Delta \mathbf{A}_{\mathbf{1}} \cdot \mathbf{T}$ & 0 & 5 & -6 & 6 & 10 & -7 & 9 & -9 & 9 & -3 & 10 \\
\hline$\Delta \mathbf{B}_{\mathbf{0}} \cdot \mathbf{T}$ & 0 & 5 & 0 & 7 & 1 & 6 & 7 & 6 & 0 & 10 & 10 \\
\hline$\Delta \mathbf{C}_{\mathbf{1}} \cdot \mathbf{X}$ & 0 & 9 & 2 & 8 & 10 & 8 & 8 & 6 & -2 & 12 & -11 \\
\hline$\Delta \mathbf{C}_{\mathbf{2}} \cdot \mathbf{X}$ & 0 & -2 & -2 & -2 & -10 & -10 & -9 & 4 & 0 & -10 & -10 \\
\hline$\Delta \mathbf{B}_{\mathbf{1}} \cdot \mathbf{U}$ & 0.0 & -1 & 11 & 2 & 11 & 6 & 11 & 6 & 5 & -5 & 8 \\
\hline$\Delta_{\mathbf{B}} \cdot \mathbf{T}$ & 0.0 & 0 & 3 & 8 & 6 & -8 & 0 & -3 & 14 & -2 & 14 \\
\hline $\boldsymbol{\Delta}_{\mathbf{B}} \cdot \mathbf{Y}$ & 0.0 & 1 & 4 & 3 & -4 & 10 & -13 & 11 & 4 & -4 & 9 \\
\hline $\mathbf{B}_{\mathbf{0}} \mathbf{Z}$ & 53 & 50 & 50 & 51.1 & 50.7 & 41.6 & 43.3 & 50.7 & 43.3 & 52.0 & 40.4 \\
\hline $\mathbf{B}_{\mathbf{1}} \cdot \mathbf{Z}$ & 55 & 60 & 55.6 & 50 & 50.1 & 59.1 & 43.3 & 49.7 & 50 & 60.0 & 41.1 \\
\hline $\mathbf{C}_{\mathbf{0}} \cdot \mathbf{Z}$ & 53 & 66.2 & 52.5 & 52.5 & 52.5 & 46.9 & 67.5 & 74.2 & 52.5 & 30.0 & 52,5 \\
\hline $\mathbf{C}_{\mathbf{1}} \cdot \mathbf{Z}$ & 53 & 70.5 & 52.5 & 51.2 & 52.5 & 57.9 & 52.5 & 60.6 & 52.5 & 75.0 & 45.8 \\
\hline $\mathbf{C}_{\mathbf{2}} \cdot \mathbf{Z}$ & 52 & 63.4 & 52.5 & 53.1 & 54.2 & 44.6 & 67.5 & 74.9 & 74.2 & 60.0 & 74.2 \\
\hline
\end{tabular}

Table A.10: Parameter values for shell on three supports in the NURBS design space (values are expressed in meters). 